\title{
Radiation, dissipation and Dufour effects on MHD free convection Casson fluid flow through a vertical oscillatory porous plate with ion-slip current
}

\author{
K.V.B. Rajakumar ${ }^{1,2 *}$, K.S. Balamurugan ${ }^{3}$, M. Umasankara Reddy ${ }^{2}$, Ch. V. Ramana Murthy ${ }^{4}$ \\ ${ }^{1}$ Research scholar in Rayalaseema University, Kurnool, (A.P), India \\ ${ }^{2}$ Department of Mathematics Kallam Haranadhareddy institute of Technology, Guntur.(A.P), India \\ ${ }^{3}$ Department of Mathematics, RVR\&JC College of Engineering, Guntur, Andhra Pradesh, India \\ ${ }^{4}$ Department of Mathematics, Sri Vasavi Institute of Engineering \& Technology, Nanadamuru (AP), India
}

Corresponding Author Email: kvbrajakumar@gmail.com

https://doi.org/10.18280/ijht.360214

Received: 8 Demember 2017

Accepted: 16 May 2018

\section{Keywords:}

Dufour, radiation absorption, viscous dissipation, hall effect, ion-slip effects, MHD, chemical reaction, perturbation law

\begin{abstract}
In this paper Dufour, Radiation absorption, Chemical reaction, and viscous dissipation effects on Unsteady Magneto Hydrodynamic free convective Casson fluid flow through a semiinfinite vertical Oscillatory porous plate of time dependent permeability with Hall and Ion-Slip Current in a Rotating System was investigated. The dimensionless governing equations for this investigation are solved analytically by using multiple regular perturbation law. The effects of different parameters on velocity, temperature and concentration fields are shown graphically. With the aid of these, the expression for the skin friction, Nusselt number and Sherwood number profiles was done with the help of tables. It was found that as the Ion-slip parameter increases the Velocity and temperature profiles decreases but the velocity and temperature decrease with increases in Hall current parameter. Dufour effect and thermal radiation increases it leads to increase in both velocity and temperature. However an increase in both chemical reactions, Schmidt number, leads to decreases in concentration.
\end{abstract}

\section{INTRODUCTION}

Non-Newtonian fluid flow arises in many branches of chemical and material processing engineering. There are different types of non-Newton fluids like Viscoelastic fluid, couple stress fluid, microplar fluid and power-law fluid etc. In addition with these, there is another non-Newtonian fluid model is known as the Casson fluid model. It is one of the pseudo plastic fluids that were introduced by Casson in 1995 . It is a part of mechanics based mostly at the time conception that a fluid particle could also be taken into thought as continuous in a very structure. The Casson fluid model is one of the non-Newtonian fluid models which reveal the characteristics of yield stress. Also Casson fluid acts like a solid when the shear stress less than the yield stress is applied, and it moves if the applied shear stress is greater than the yield stress. This has significant applications in polymer processing industries and biomechanics. The examples of Casson fluid are of the type are as follows: jelly, tomato sauce, honey, soup, concentrated fruit juices, gypsum paste, cream, Aloe era juices etc. Biswas et al. [1] unsteady heat and mass transfer of Casson fluid flow past a vertical plate in presence of radiation and chemical reaction has been studied. From this paper it was found that Non-dimensional partial differential equations are solved by using explicit finite difference method. The study of Casson fluid flow particularly in the Magneto-Hydrodynamic effect with free convection flow has been received considerable attention to many researchers in last few years. In addition, Magneto Hydrodynamic is the branch of continuum mechanics which deals with the flow of electrically conducting fluid in electrical and magnetic fields. The flow of an electrically conducting fluid in the presence of a magnetic field is important in various area of technology. There are many applications of Magneto Hydrodynamic free convection flows in fiber and granular insulation, geothermal systems etc. Throughout the last few decades a great deal of research work have been done about the magneto-hydrodynamic effects of Casson fluid flow, Hall and Ion current, Dufour, Radiation absorption, viscous dissipation, and Chemical reaction through porous plate.

In fluid mechanics, destruction of fluctuating velocity gradients due to viscous stresses is known as viscous dissipation. This partial irreversible process is often referred to as transformation of kinetic energy into internal energy of the fluid. Jithender Reddy et al. [2] in this paper, an unsteady Magneto Hydrodynamic natural convection, heat transfer electrically conductive non-Newtonian Casson fluid over an oscillating vertical porous plate taken in to the account with an influence of viscous dissipation. Here it was found that the fluid velocity increased as increasing of Grashof number, Porous medium and Eckert number while decreased as increasing of Casson fluid parameter, Magnetic number and Phase angle. The local skin-friction coefficient increased with increasing of Prandtl number, Magnetic parameter while decreased with increasing Grashof number, Casson parameter, porous medium, and time and Eckert number. Hazem Ali Attia et al. [3] reported that the unsteady Magneto Hydrodynamic flow of an electrically conducting viscous incompressible nonNewtonian Casson fluid bounded by two parallel nonconducting porous plates in presence of viscous dissipation effect were studied with heat transfer considering the Hall Effect. In this article it was observed that an external uniform magnetic field was applied which is perpendicular to the plates and the fluid motion is subjected to a uniform suction and 
injection. The lower plate is stationary and the upper plate is suddenly set into motion and simultaneously suddenly isothermally heated to a temperature other than the lower plate temperature. The governing equations were solved by using finite element method. Srinivasa Raju et al [4]. Consider influence of a viscous dissipative, chemical reaction on an unsteady Magneto Hydrodynamic free convective, Casson fluid flow over a vertically inclined plate in presence of magnetic field, heat and mass transfer. In this research Hall and Ion-slip current was not consider. Saidulu et al. [5] portraying the boundary layer flow of a non-Newtonian Casson fluid accompanied by heat transfer towards a porous exponentially stretching sheet with velocity slip and thermal slip conditions in the presence of thermal radiation, suction/blowing, viscous dissipation and heat source/sink effects. In this examination Hall and Ion-slip current was not consider. R. Srinivasa Raju et al. [6] Unsteady Magneto Hydrodynamic natural convective, heat and mass transfer, electrically conducting Casson fluid flow over on an vertical surface taken in to the account with angle of inclination, chemical reaction, viscous dissipation and constant heat flux. The governing non-linear partial differential equations are solved by using finite element method. In this paper it was observed that the velocity decreases with an increase in magnetic field parameter, Schmidt number, chemical reaction parameter, Casson fluid parameter, angle of inclination parameter and Prandtl number while it increases with an increase in Eckert number and Soret number. Temperature increases with an increase in Eckert number while it decreases with increasing values of Prandtl number. Concentration increases with increase in Soret number while it decreases with an increase in Schmidt number and chemical reaction parameter.

Ion-slip and Hall current are probably going to be fundamental in flows of lab plasma when a solid magnetic field of a uniform quality is connected and drawn the consideration of the analysts because of their differed hugeness in fluid metals electrolytes arrive ionized gasses. The Hall Effect is the having of a voltage effect over an electrical conductor, transverse to an electric current in the transmitter and an electromagnetic field is opposite to the current. It was found by Edwin Hall et al. [7]. The present advancement of Magneto Hydrodynamic application is toward a solid magnetic field and toward a low thickness of the gas. Under this condition, the Hall current ends up noticeably critical. That significance considered by numerous analysts. N.Nowroz Anika et al. [8]. The effect of Hall, Ion slip over an infinite vertical plate for microplar fluid within the magnetic field has been investigated. The governing equations are solved by using finite difference technique. Srinivasacharya et al. [9] analyzed the significance of hall and ion-slip parameter on steady mixed convective heat transfer flow of a nanofluid in a vertical channel. The homotopy analysis method was utilized to solve the nonlinear ordinary differential conditions. From this paper it was observed that as Hall parameter builds it prompts the increments in velocity and temperature, yet the actuated flow velocity and Nano particle concentration diminished. As Ion-slip parameter builds it prompts increments in the velocity and temperature, however the actuated flow velocity and Nano particle concentration diminishes. Srinivasacharya et al. [10]. Analyzed the significance of Hall and Ion-slip parameter on steady mixed convective heat transfer flow of a nanofluid in a vertical channel. The HAM is used to solve the nonlinear ordinary differential equations. In perspective of the above examination, viscous dissipation effects were not considered on Casson fluid models on an oscillating vertical plate. K. Pushpalatha et al. [11] unsteady free convection flow of a Casson fluid bounded by a moving vertical flat plate in a rotating system with convective boundary conditions. The non-dimensional equations are solved by using perturbation technique. In this investigation Hall and Ion-slip current were not consider.

The energy flux caused due to composition gradient is known as Diffusion thermo effect or Dufour effect. The experimental study on diffusion thermo effect on heat transfer allied problems was first performed by L.dufour in 1872 . Temperature gradients can also generate mass fluxes and is known as thermo-diffusion effect .usually, the thermal diffusion is the occurrence of a diffusion flux due to a temperature gradient. The Dufour effect is the reciprocal phenomenon, the occurrence of a heat flux due to a chemical potential gradient. Both effects have been extensively studied in gases, and the Soret. Consolidated effects have been contemplated both hypothetically and tentatively in fluids. Be that as it may, incompletely due to the littleness of the effect, exact estimations of the Dufour effect in fluids have as it were. Pannerselvi et al. [12]. Contemplated two dimensional unsteady free convection and mass transfer flow and viscous fluid past an infinite vertical porous plate of time dependent permeability and heat source with oscillatory section velocity in the presence of Dufour effect, Hall Effect and Ion slip effect was researched. A uniform magnetic field is applied normal to the direction of the flow. The non dimensional representing conditions are explained numerically by utilizing perturbation technique. Abuga et al. [13] the influence of hall effect, rotating system, thermal diffusion and diffusion thermo on magneto hydrodynamic fluid flow past an infinite moving vertical plate with externally heating and cooling of the plate in case of isothermal and ramped wall temperature was investigated. In this investigation the governing equations was solved by finite element free Galerkin method. Odelu Ojjela et al. [14]. Investigated the Hall and ion slip current on free convection flow, heat and mass transform of an electrically conducting couple stretch fluid through permeable channels with chemical reaction, Dufour and Soret effects. In the above examination hall and ion effect was not consider on Casson fluid models on an oscillating vertical plate.

The effects of radiation on Magneto Hydrodynamic flow and heat transfer issues have turned out to be modernly more critical. A few engineering forms occur at high temperatures and henceforth the information of thermal radiation heat transfer is fundamental for planning of legitimate supplies, for example, gas turbines, atomic power plants and diverse impetus gadgets for air ship, rockets and satellites. At the point when radiative heat move happens in the electrically conducting fluid, it is ionized because of the high working temperature. In perspective of these, numerous analysts have made commitments to the investigation of fluid flow with thermal radiation. Hari et al. [15]. studied unsteady free convective Magneto Hydrodynamic boundary layer Casson fluid flow past an oscillating vertical plate implanted through porous medium within the sight of uniform transverse attractive field, thermal radiation and synthetic response. Shateyi et al. [16]. Investigated the effects of thermal radiation, Hall currents, and Soret and Dufour effects on Magneto Hydrodynamic mixed convection flow over a vertical surface in porous media, from this paper it was 
observed that the fluid temperature increases due to an increase in the thermal radiation. Also they found that the concentration decreases as the radiation parameter value is increased. Sreedevi et al. [17] reported that the radiation absorption, variable viscosity, Hall current of a Magneto Hydrodynamic free-convective flow and heat and mass transfer over a stretching sheet within the heat generation or absorption. Gireesha et al. [18]. Analyzed the effect of radiation on unsteady laminar flow with heat and mass transfer of an electrically conducting, chemically reactive viscoelastic fluid in irregular channel with subject to convective boundary condition has been investigated. The perturbation technique is used to solve governing coupled nonlinear partial differential equations. In the above investigation Casson fluid model were not discussed. Delowar et al. [19] analyzed Unsteady Magneto Hydrodynamic free convection and mass transfer flow through a vertical oscillatory porous plate with hall, ion-slip currents and heat source in a rotating system. In this paper it was observed that the governing equations of the problem contain a system of non-linear coupled partial differential equations. The coupled non-linear partial differential equations are solved by explicit finite difference method.

Chemical reaction in the heat and mass transfer problems has been received a substantial quantity of attention in recent years. In processes equivalent to drying, evaporation at the surface of water body, energy transfer in wet cooling, and also the flow in a very desert cooler, heat and mass transfer occur at the same time. Chemical reaction may be statute as either uniform or hetero geneous processes. A uniform rection is one that occurs uniformly through a given section. In distinction, a heterogeneous reaction takes place in a very restricted region or among the boundary of a section. A reaction is claimed to be initial order, if the rate of reaction is directly proportional to the concentration itself that has sever-al applications in numerous chemical engineering processes and different industrial applications equivalent to chemical compound production, producing of ceramics or tableware, and food process. Mythili et al. [20] studied chemically reacting Casson fluid flow over a vertical cone and flat plate saturated with non-Darcy porous medium in presence of heat absorbing. Jasmine Benazir et al. [21] considered the effects of double dispersion, non-uniform heat source/sink and higher order chemical reaction on unsteady, free convective, Magneto Hydrodynamic Casson fluid flow over a vertical cone and flat plate saturated with porous medium. The extensively validated and unconditionally stable numerical solutions are obtained for the governing equations of two dimensional boundary layer flows by using the finite difference scheme of CrankNicolson type. R. Srinivasa Raju [22] analyzed the effects of chemical reaction and combined buoyancy effects on an unsteady Magneto Hydrodynamic mixed convective flow along an infinite vertical porous plate in the presence of hall current. Salem and Abd El-Aziz [23] have reported the effect of hall currents and chemical reaction on Hydro Magnetic flow of a stretching vertical surface with internal heat generation or absorption. Sudhakar et al. [24] studied the effect of hall current on an unsteady Magneto Hydrodynamic flow along a porous flat plate with thermal diffusion, dif- fusion thermo, and chemical reaction by using Galerkin finite element method. Srinivasa Raju [25] considered the combined effects of thermal-diffusion and diffusion-thermo on unsteady free convection fluid flow past an infinite vertical porous plate in presence of magnetic field and chemical reaction using finite element technique. Srinivasa Raju et al. [26] reported that the applications of finite element method to unsteady Magneto Hydrodynamic free convection flow past a vertically inclined porous plate including thermal diffusion and diffusion thermo effects. Srinivasa Raju et al. [27] found both analytical and numerical results of unsteady Magneto Hydrodynamic free convective flow past an exponentially moving vertical plate with heat absorption and chemical reaction.

Remembering every one of these realities in this work the effect the Dufour, Radiation absorption, Chemical reaction, and viscous dissipation effects on Unsteady Magneto Hydrodynamic free convective Casson fluid flow through a semi-infinite vertical Oscillatory porous plate of time dependent permeability with Hall and Ion-Slip Current in a Rotating System was investigated. The perturbation technique is employed to solve governing coupled non-liner partial differential equations.

\section{MATHEMATICAL FORMULATION}

The unsteady flow of a electrically conducting incompressible viscous fluid past semi-infinite vertical porous plate $\mathrm{y}=0$ has been considered, with the $\mathrm{x}$-axis chosen along the plat, when the plate velocity $U(t)$ oscillates in $t$ with a frequency $n$ and is given as $\mathrm{U}(\mathrm{t})=\mathrm{U}_{0}(1+$ connt $)$.

The constitutive equation for the Casson fluid [11] can be written as

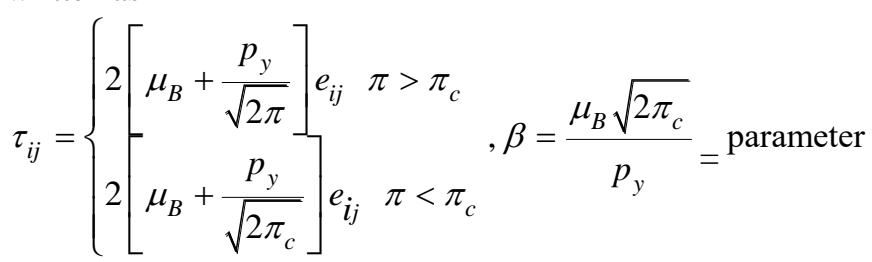

of the Casson fluid $\pi=e_{i j} e_{i j}$ and $e_{i j}$ is the $(i, j)^{t h}$ component of deformation rate. It is the critical value of this product based on the non-Newtonian model, $\pi$ is the product based on the non-Newtonian fluid, $\pi_{C}$ is a critical value of this product, $\mu_{B}$ is plastic dynamic viscosity of the non-Newtonian fluid.

$p_{y}$ is the yield tress of the fluid.

- Let the $\mathrm{x}^{*}$-axis be brought the permeable plate the upward way and $y^{*}$-axis toward the path opposite to the flow.

- Let the $x^{*}$ and $y^{*}$ are the dimensional distance along the perpendicular to the plate and $t *$ is the time. The physical model of the flow problem is shown in Figure A: $u^{*}$ and $\mathrm{V}^{*}$ are the components of dimensional velocities along $\mathrm{x}^{*}$ and $\mathrm{y}^{*}$ directions. $\mathrm{C}^{*}$ and $\mathrm{g}^{*}$ are the dimensional concentration, acceleration due to gravity, $Q_{0}\left(T^{*}-T_{\infty}^{*}\right)$ is the amount of heat generated per unit volume. $Q_{0}$ Is the constant where either $Q_{0}<0$ or $Q_{0}>0$, g is acceleration due to gravity. $g \beta\left(T^{*}-T_{\infty}^{*}\right)$ is the body force due to nonuniform temperature, $g \beta^{*}\left(C^{*}-C_{\infty}^{*}\right)$ is the body force due to non uniform concentration the flow is assumed to be in $\mathrm{x}$-direction and which is taken along the plate in upward direction and $y$-axis is normal to it. Initially the fluids as well as the plate are at rest but for time $t>0$ the whole system is allowed to rotate with a constant angular velocity $\Omega$ about the y-axis. In addition, it is assumed that the temperature and Concentration at the wall as well as the suction velocity are exponentially varying with time.

- Assumed transverse magnetic field of the uniform strength $\mathrm{B}_{0}$ is to be utilizable normal to the plate. 
- The radiate heat flux in the $\mathrm{x}^{*}$ - direction is considered negligible in comparison with that in $\mathrm{y}^{*}$ - direction.

- It is assumed that voltage is not applied which implies the absence of an electric field.

- The heat source, Hall Effect, Ion-slip effect and Dufour effects are considered in the view of relatively high electron-atom collision frequency.

- Induced magnetic field is assumed to be negligible because of the magnetic Reynolds number of a partially-ionized fluid is very small.

- Viscous dissipation, Radiation absorption and Darcy resistance terms are taken into account the constant permeability porous medium. Since the plate is semiinfinite in length the entire flow variables are functions of $\mathrm{y}$ and $\mathrm{t}$ only.

- The fluid has constant thermal conductivity and kinematic viscosity, the Boussinesqu's approximation has been taken for the flow.

- The homogeneous chemical reaction is of first order with rate constant $\mathrm{K}_{\mathrm{r}}$ between the diffusing species and the fluid is considered.

- The physical configuration of the problem is shown in the figure A: A uniform magnetic field $B$ is acting transverse the plate. Using the relation $\nabla \cdot \mathrm{B}=0$ or the magnetic field $\mathrm{B}=\left(\mathrm{B}_{\mathrm{x}}, \mathrm{B}_{\mathrm{y}}, \mathrm{B}_{\mathrm{z}}\right), \mathrm{B}_{\mathrm{y}}=\mathrm{B}_{0}$ has been consider everywhere in the fluid ( $B_{0}$ is a constant). If $J=\left(J_{x}, J_{y}, J_{z}\right)$ is the current density, from the relation $\nabla . \mathrm{J}=0, \mathrm{~J}_{\mathrm{y}}=$ constant has been obtained. Since the plate is electrically non-conduction, $\mathrm{J}_{\mathrm{y}}=0$ at the plate and hence zero everywhere.

- When the strength of magnetic field is very large, the generalized Ohm's law for the current in the form:

$$
\overline{\mathrm{J}}=\sigma\left(1+\left(\omega\left(\mathrm{J}_{\mathrm{e}}\right)^{-1}\right)^{2}\right)^{-1}\left(\overline{\mathrm{E}}+(\overline{\mathrm{V}} \times \overline{\mathrm{B}})-\left(\mathrm{en}_{\mathrm{e}}\right)^{-1}(\overline{\mathrm{J}} \times \overline{\mathrm{B}})\right)
$$

where $\omega$ is the electron cyclotron and $\vartheta_{e}$ is the electron-atom collision frequency. When the ratio $\omega\left(\vartheta_{e}\right)^{-1}$ is very large, the phenomenon is called" Ion-slip."

Hence dimensional governing equations are:

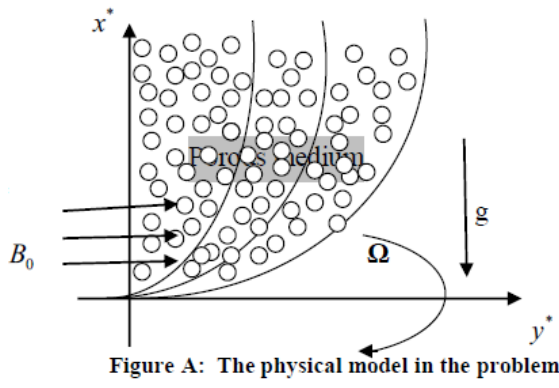

Equation of Momentum:

$$
\left.\begin{array}{r}
{\left[\frac{\partial u^{*}}{\partial \tau^{*}}\right]=\vartheta\left[1+\frac{1}{\beta}\right]\left[\frac{\partial^{2} u^{*}}{\partial y^{*^{2}}}\right]+g \psi\left(T^{*}-T_{\infty}^{*}\right)} \\
+g \psi^{*}\left(C^{*}-C_{\infty}^{*}\right)+2 \Omega w^{*}-\frac{\vartheta}{k}\left[1+\frac{1}{\beta}\right]\left[u^{*}\right] \\
-\frac{B_{0}^{2} \sigma_{e}\left[\alpha_{e} u^{*}+\beta_{e} w^{*}\right]}{\rho\left[\alpha_{e}^{2}+\beta_{e}^{2}\right]}
\end{array}\right\}
$$

$$
\left.\begin{array}{rl}
{\left[\frac{\partial w^{*}}{\partial \tau^{*}}\right]=\vartheta\left[1+\frac{1}{\beta}\right]\left[\frac{\partial^{2} w^{*}}{\partial *^{2}}\right]-\frac{\vartheta}{k^{*}}\left[1+\frac{1}{\beta}\right]\left[w^{*}\right]} \\
-2 \Omega\left[u^{*}\right]+\frac{B_{0}^{2} \sigma_{e}\left[\beta_{e} u^{*}-\alpha_{e} w^{*}\right]}{\rho\left[\alpha_{e}^{2}+\beta_{e}^{2}\right]}
\end{array}\right\}
$$

Equation of Energy:

$$
\begin{aligned}
& {\left[\frac{\partial T^{*}}{\partial \tau^{*}}\right]=\frac{K}{\rho C_{p}}\left[\frac{\partial^{2} T^{*}}{\partial y^{*^{2}}}\right]+\frac{D_{m} K_{T}}{C_{S} C_{P}}\left[\frac{\partial^{2} C^{*}}{\partial y^{*^{2}}}\right]} \\
& +\frac{Q_{0}}{\rho C_{p}}\left[T^{*}-T_{\infty}^{*}\right]-\frac{1}{k \rho C_{p}}\left[\frac{\partial q_{r}^{*}}{\partial y^{*}}\right]+R^{*}\left[C^{*}-C_{\infty}^{*}\right] \\
& +\frac{\vartheta}{\rho C_{p}}\left[1+\frac{1}{\beta}\right]\left[\left(\frac{\partial u^{*}}{\partial y^{*}}\right)^{2}+\left(\frac{\partial w^{*}}{\partial y^{*}}\right)^{2}\right]
\end{aligned}
$$

Concentration species diffusion equation:

$$
\left[\frac{\partial C^{*}}{\partial \tau^{*}}\right]=D_{m}\left[\frac{\partial^{2} C^{*}}{\partial y^{*^{2}}}\right]-K_{r}\left[C^{*}-C_{\infty}^{*}\right]
$$

The initial and boundary conditions are as

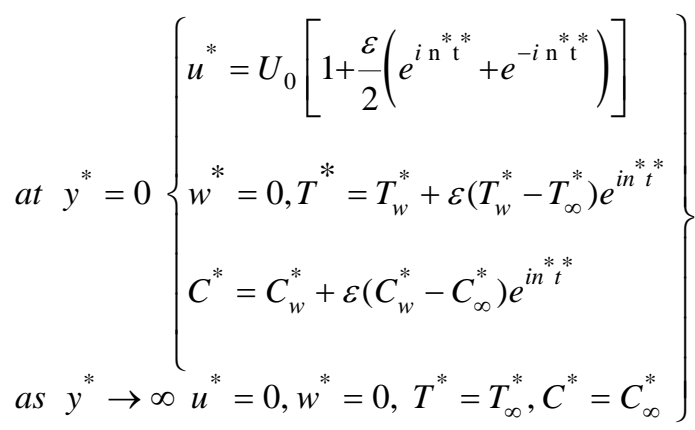

Using the relation in the radiative heat flux $\left(q_{r}\right)$ for the optically thin non gray gas near equilibrium is given by

$$
\frac{\partial q_{r}}{\partial y}=4 I^{1} T\left[T-T_{1}\right], \quad I^{1}=\int_{0}^{\infty} K_{\lambda_{1} w}\left[\frac{\partial e_{b \lambda_{1}}}{\partial T}\right] d \lambda_{1}
$$

$K_{\lambda_{1} w}$ is the radiation absorption coefficient at the wall and $e_{b \lambda_{1}}$ is the Plank's constant.

The permeability of the porous medium is assumed to be of the following:

$$
k^{*}=k_{0}\left[1+\varepsilon e^{-n t}\right]
$$

$k_{0}$ is the constant permeability of the medium.

Introducing the following non-dimensional quantities in the equation (2), (3), (4) and (5) 


$$
\left.\begin{array}{c}
u=\frac{u^{*}}{U_{0}}, w=\frac{w^{*}}{U_{0}} y=\frac{y^{*} U_{0}}{\vartheta}, t=\frac{U_{0}^{2} \tau^{*}}{\vartheta} \\
n=\frac{\vartheta_{n}^{*}}{U_{0}{ }^{2}}, \frac{T^{*}-T_{\infty}^{*}}{T_{w}^{*}-T_{\infty}^{*}}=\theta, \frac{C^{*}-C_{\infty}^{*}}{C_{w}^{*}-C_{\infty}^{*}}=\phi
\end{array}\right\}
$$

Then the equation (2), (3), (4) and (5) reduce to the following non- dimensional form of equations

$$
\left.\begin{array}{r}
{\left[\frac{\partial u}{\partial t}\right]=\left[1+\frac{1}{\beta}\right]\left[\frac{\partial^{2} u}{\partial y^{2}}\right]+G_{r}[\theta]+G_{m}[\phi]+2 R[w]} \\
-\gamma\left[1+\frac{1}{\beta}\right][u]-\frac{M\left[\alpha_{e} u+\beta_{e} w\right]}{\left[\alpha_{e}^{2}+\beta_{e}^{2}\right]}
\end{array}\right\}
$$

$$
\left.\begin{array}{r}
{\left[\frac{\partial w}{\partial t}\right]=\left[1+\frac{1}{\beta}\right]\left[\frac{\partial^{2} w}{\partial y^{2}}\right]-2 R[u]-\gamma\left[1+\frac{1}{\beta}\right][w]} \\
+\frac{M\left[\beta_{e} u-\alpha_{e} w\right]}{\left[\alpha_{e}^{2}+\beta_{e}^{2}\right]}
\end{array}\right\}
$$

$$
\left.\begin{array}{r}
{\left[\frac{\partial \theta}{\partial t}\right]=\frac{1}{\operatorname{Pr}}\left[\frac{\partial^{2} \theta}{\partial y^{2}}\right]-N[\theta]+\operatorname{Dr}\left[\frac{\partial^{2} \phi}{\partial y^{2}}\right]} \\
+E c\left[1+\frac{1}{\beta}\right]\left[\frac{\partial F}{\partial y} \frac{\partial \bar{F}}{\partial y}\right]^{2}+R_{a}[\phi]
\end{array}\right\}
$$

$$
\left[\frac{\partial \phi}{\partial t}\right]=(S c)^{-1}\left[\frac{\partial^{2} \phi}{\partial y^{2}}\right]-K_{r}[\phi]
$$

$\xi=\frac{\vartheta Q_{0}}{\rho U_{0}^{2} C_{P}}, M=\frac{\sigma_{e} B_{0}^{2} \vartheta}{\rho U_{0}^{2}}, G_{m}=\frac{\vartheta \psi^{*} g\left[C_{w}^{*}-C_{\infty}^{*}\right]}{U_{0}^{2}}$

$G_{r}=\frac{\vartheta \psi g\left[T_{w}^{*}-T_{\infty}^{*}\right]}{U_{0}^{2}}, \eta=\frac{4 \vartheta I^{\prime}}{K_{p} C_{p} U_{0}^{2}}, R=\frac{\Omega \vartheta}{U_{0}^{2}}$

$S c=\frac{\vartheta}{D_{m}}, R_{a}=\frac{R^{*} \vartheta\left[C_{w}^{*}-C_{\infty}^{*}\right]}{U_{0}^{2}\left[T_{w}^{*}-T_{\infty}^{*}\right]}, \operatorname{Pr}=\frac{\rho \vartheta C_{p}}{\sigma}$

$D r=\frac{D_{m} K_{T}\left[C_{w}^{*}-C_{\infty}^{*}\right]}{\vartheta C_{S} C_{P}\left[T_{w}^{*}-T_{\infty}^{*}\right]}, \alpha_{e}=1+\beta_{e} \beta_{i}, \gamma=\frac{\vartheta^{2}}{k^{*} U_{0}^{2}}$

$E c=\frac{U_{0}^{2}}{C_{p}\left[T_{w}^{*}-T_{\infty}^{*}\right]}, K_{r}=\frac{k_{1} \vartheta}{V_{0}^{2}}, N=[\xi+\eta]$

$\lambda=\left[\left[2 R i+\gamma\left[1+\frac{1}{\beta}\right]\right]+\frac{M\left[-\alpha_{e}+i \beta_{e}\right]}{\left[\alpha_{e}^{2}+\beta_{e}^{2}\right]}\right]$

Equations (11) and (12) are displayed, in a reduced form, as

$$
\frac{\partial U}{\partial t}=\left[1+\frac{1}{\beta}\right] \frac{\partial^{2} U}{\partial y^{2}}+G_{r} \theta+G_{m} \phi-\lambda F
$$

$$
\left.\begin{array}{c}
U=\left[1+\frac{\varepsilon}{2}\left(e^{i \mathrm{nt}}+e^{-i \mathrm{nt}}\right)\right]=1 \\
\left.\begin{array}{c}
\theta=1+\varepsilon e^{i n t}, \phi=1+\varepsilon e^{i n t} \\
U \rightarrow 0, \theta \rightarrow 0, \phi \rightarrow 0
\end{array}\right\} \text { as } y \rightarrow \infty
\end{array}\right\}
$$

\section{METHOD OF SOLUTION}

The resulting system of nonlinear ODEs Esq. (12), (13) and (15) subject to the boundary conditions presented in Esq. (16) has been explored numerically through Multiple Regular Perturbation law. This is more economical and flexible from analytical point of view. Finding multiple regular perturbation method is an art rather than a science. In research it is useful to be responsive to suggestions from the physics. There is certainly no routine method appropriate to all problem, or even classes of problems. Instead one needs a determination to exploit the smallness of the parameter. Obtaining good numerical values for the solution is not the only quest of a perturbation method. One can hope that the analysis will reveal some physical insights through the simplified physics of the limiting problem. The behaviors of velocity, temperature, concentration, Skin-friction coefficient, Nusselt number and Sherwood numbers has been discussed in detail for variation of thermo physical parameters. The solution is assumed to be as:

$$
\left.\begin{array}{l}
U=U_{0}[y]+\varepsilon e^{i n t} U_{1}[y]+o\left[\varepsilon^{2}\right] \ldots . \\
\theta=\theta_{0}[y]+\varepsilon e^{i n t} \theta_{1}[y]+o\left[\varepsilon^{2}\right] \ldots . . \\
\phi=\phi_{0}[y]+\varepsilon e^{i n t} \phi_{1}[y]+o\left[\varepsilon^{2}\right] \ldots \ldots .
\end{array}\right\}
$$

After Substitute (17) in the equations (12), (13) and (15) then we get

$$
\begin{aligned}
& {\left[1+\frac{1}{\beta}\right] U_{0}^{\prime \prime}-\lambda U_{0}=-G_{r} \theta_{0}-G_{m} \phi_{0}} \\
& {\left[1+\frac{1}{\beta}\right] U_{1}^{\prime \prime}-[\lambda+n i] U_{1}=-G_{r} \theta_{1}-G_{m} \phi_{1}}
\end{aligned}
$$

$$
\left.\begin{array}{rl}
\theta_{1}^{\prime \prime}-\operatorname{Pr}[N+i n] \theta_{1} & =-\operatorname{Pr} \operatorname{Dr} \phi_{1}^{\prime \prime}-\operatorname{Pr} R_{a} \phi_{1} \\
& -2 \operatorname{Pr} E c\left[1+\frac{1}{\beta}\right] U_{0}^{\prime} U_{1}^{\prime}
\end{array}\right\}
$$

$$
\left.\begin{array}{r}
\theta_{0}^{\prime \prime}-\operatorname{Pr} N \theta_{0}=-\operatorname{Pr} \operatorname{Dr} \phi_{0}^{\prime \prime}-\operatorname{Pr} E c\left[1+\frac{1}{\beta}\right]\left[U_{0}^{\prime}\right]^{2} \\
-\operatorname{Pr} \operatorname{Ra\phi _{0}}
\end{array}\right\}
$$

$\phi_{0}^{\prime \prime}-S_{c} K_{r} \phi_{0}=0$

$\phi_{1}^{\prime \prime}-S_{c}\left[K_{r}+n\right] \phi_{1}=0$

The corresponding boundary conditions can be written as 


$$
\begin{aligned}
& \left.\begin{array}{r}
U_{0}=1, U_{1}=0, \theta_{0}=1 \theta_{1}=1, \\
\phi_{0}=1, \phi_{1}=1
\end{array}\right\} \text { at } y=0 \\
& \left.\left.\begin{array}{rl}
U_{0}=0, U_{1}=0, \theta_{0} & \rightarrow 0, \theta_{1} \rightarrow 0 \\
\phi_{0} & \rightarrow 0, \phi_{1} \rightarrow 0,
\end{array}\right\} \text { as } y \rightarrow \infty\right\}
\end{aligned}
$$

Here primes denote differentiation w.r.t. y the equations (18)-(21) are still coupled and non-linear, whose exact solutions are not possible. So we expand $U_{0}, U_{1}, \theta_{0}, \theta_{1}$.

First we solve equation (22) and equation (23) by using equation (24). Then we get

$\phi_{0}=e^{-(\sqrt{S c K r}) y}$

$\phi_{1}=e^{-(\sqrt{S c(K r+n)}) y}$

Now using multi parameter perturbation technique and assuming $E c<<1$.

$$
\left.\begin{array}{l}
U_{0}=U_{00}+E c U_{01}+0[\varepsilon]^{2} \ldots \ldots . . \\
\theta_{0}=\theta_{00}+E c \theta_{01}+0[\varepsilon]^{2} \ldots \ldots . \\
U_{1}=U_{10}+E c U_{11}+0[\varepsilon]^{2} \ldots \ldots \ldots \\
\theta_{1}=\theta_{10}+E c \theta_{11}+0[\varepsilon]^{2} \ldots \ldots \ldots
\end{array}\right\}
$$

By using equations (27) in equations (18)-(21) and equating the coefficients of like powers of Ec neglecting those of $[E c]^{2}$ and $0[\varepsilon]^{2}$ we get the following set of differential equation

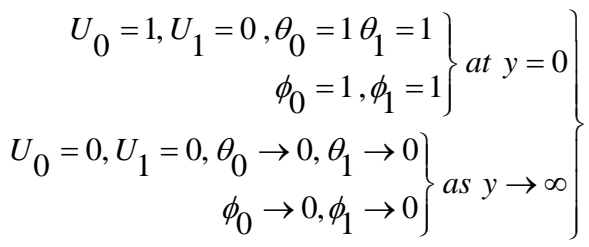

$\left[1+\frac{1}{\beta}\right] U_{00}^{\prime \prime}-\lambda U_{00}=-G_{r} \theta_{00}-G_{m} \phi_{0}$

$\left[1+\frac{1}{\beta}\right] U_{01}^{\prime \prime}-\lambda U_{01}=-G_{r} \theta_{01}$

$\left[1+\frac{1}{\beta}\right] U_{10}^{\prime \prime}-[\lambda+n i] U_{10}=-G_{r} \theta_{10}-G_{m} \phi_{1}$

$\left[1+\frac{1}{\beta}\right] U_{11}^{\prime \prime}-[\lambda+n i] U_{11}=-G_{r} \theta_{11}$

$\theta_{10}^{\prime \prime}-\operatorname{Pr}[N+i n] \theta_{10}=-R_{a} \phi_{1} \operatorname{Pr}-\operatorname{Pr} \operatorname{Dr} \phi_{1}{ }^{\prime \prime}$

$$
\theta_{11}^{\prime \prime}-\operatorname{Pr}[N+i n] \theta_{11}=-2 \operatorname{Pr}\left[1+\frac{1}{\beta}\right] U_{00}^{\prime} U_{10}^{\prime}
$$

$$
\begin{aligned}
& \theta_{00}^{\prime \prime}-\operatorname{Pr} N \theta_{00}=-\operatorname{Pr} R_{a} \phi_{0}-\operatorname{Pr} \operatorname{Dr} \phi_{0}^{\prime \prime} \\
& \theta_{01}^{\prime \prime}-\operatorname{Pr} N \theta_{01}=-\operatorname{Pr}\left[1+\frac{1}{\beta}\right]\left[U_{00}^{\prime}\right]^{2}
\end{aligned}
$$

$$
\begin{aligned}
& \text { at } y=0:\left\{\begin{array}{c}
U_{00}=1, U_{01}=0, U_{10}=0, U_{11}=0, \\
\theta_{00}=1, \theta_{01}=0, \theta_{10}=1, \theta_{11}=0
\end{array}\right. \\
& \text { As } y \rightarrow \infty\left\{\begin{array}{c}
U_{00}=0, U_{01}=0, U_{10}=0, U_{11}=0, \\
\theta_{00}=0, \theta_{01}=0, \theta_{10}=0, \theta_{11}=0
\end{array}\right\}
\end{aligned}
$$

Solve Equations (29)-(36) subject to boundary conditions by using (37) we get

$$
\begin{aligned}
& U_{00}=N_{3} e^{-(\sqrt{N \mathrm{Pr}}) y}+N_{4} e^{-(\sqrt{S c K r}) y}+A_{1} e^{-p y} \\
& U_{01}=\left(\begin{array}{l}
N_{11} e^{-(\sqrt{N \mathrm{Pr}}) y}+N_{12} e^{-(2 \lambda) y}+N_{13} e^{-(2 \sqrt{N \mathrm{Pr}}) y} \\
+N_{14} e^{-2 \sqrt{S c K r} y}+N_{15} e^{-((\sqrt{N \mathrm{Pr}})+\lambda) y} \\
+N_{16} e^{-(\sqrt{S c K r}+(\sqrt{N \mathrm{Pr}})) y} \\
+N_{17} e^{-(\lambda+\sqrt{S c K r}) y}+A_{3} e^{-p y}
\end{array}\right) \\
& U_{10}=N_{18} e^{-(\sqrt{\operatorname{Pr}(N+n i)}) y}+N_{19} e^{-(\sqrt{S \mathrm{r}(K r+n)}) y}+A_{4} e^{-q y}
\end{aligned}
$$

$$
U_{11}=\left(\begin{array}{l}
N_{29} e^{-q y}+N_{30} e^{-(\sqrt{\lambda}+\sqrt{\operatorname{Pr}(N+n i)}) y} \\
+N_{31} e^{-(\sqrt{S c(K r+n)}+\sqrt{\lambda}) y}+N_{32} e^{-(\sqrt{\operatorname{Pr} N}+q) y} \\
+N_{33} e^{-(\sqrt{\operatorname{Pr} N}+\sqrt{\operatorname{Pr}(N+n i)}) y}+N_{34} e^{-(\sqrt{\operatorname{Pr} N}+\sqrt{S c(K r+n)}) y} \\
+N_{35} e^{-(\sqrt{S c K r}+q) y}+N_{36} e^{-(\sqrt{S c K r}+\sqrt{S c(K r+n)}) y} \\
+N_{37} e^{-(\sqrt{S c K r}+\sqrt{\operatorname{Pr}(N+n i)}) y}+N_{38} e^{-(\sqrt{\operatorname{Pr}(N+n i)}) y} \\
\quad+A_{6} e^{-q y}
\end{array}\right)
$$

$$
\theta_{00}=\left(1-N_{1}\right) e^{-(\sqrt{\operatorname{Pr} N}) y}+N_{1} e^{-(\sqrt{S c K r}) y}
$$

$$
\theta_{01}=\left(\begin{array}{l}
N_{5} e^{-(2 \sqrt{\lambda}) y}+N_{6} e^{-(2 \sqrt{\operatorname{Pr} N}) y}+N_{7} e^{-(2 \sqrt{S c K r}) y} \\
+N_{8} e^{-(\lambda+\sqrt{\operatorname{Pr} N}) y}+N_{9} e^{-(\sqrt{\operatorname{Pr} N}+\sqrt{S c K r}) y} \\
+N_{10} e^{-(\lambda+\sqrt{S c K r}) y}+A_{2} e^{-\sqrt{\operatorname{Pr} N} y}
\end{array}\right)
$$

$$
\theta_{10}=\left(1-N_{2}\right) e^{-\sqrt{\operatorname{Pr}(N+n i)} y}+N_{2} e^{-\sqrt{S c(K r+n)} y}
$$




$$
\theta_{11}=\left(\begin{array}{l}
N_{20} e^{-(\sqrt{\lambda}+\sqrt{\lambda+n i}) y}+N_{21} e^{-(\sqrt{\lambda}+\sqrt{\operatorname{Pr}(N+n i)}) y} \\
+N_{22} e^{-(\sqrt{\lambda}+\sqrt{S c(K r+n)}) y}+N_{23} e^{-(\sqrt{\operatorname{Pr} N}+\sqrt{\lambda+n i}) y} \\
+N_{24} e^{-(\sqrt{\operatorname{Pr} N}+\sqrt{\operatorname{Pr}(N+n i)}) y}+N_{25} e^{-(\sqrt{\operatorname{Pr} N}+\sqrt{S c(K r+n)}) y} \\
+N_{26} e^{-(\sqrt{S c K r}+\sqrt{\lambda+n i}) y}+N_{27} e^{-(\sqrt{S c K r}+\sqrt{S c(K r+n)}) y} \\
+N_{28} e^{-(\sqrt{S c K r}+\sqrt{\operatorname{Pr}(N+n i)}) y}+A_{5} e^{-\sqrt{\operatorname{Pr}(N+n i)} y}
\end{array}\right)
$$

\subsection{Velocity (F), Temperature $(\theta)$ and Concentration $(\phi)$}

$$
\theta=\left\{\begin{array}{l}
\left(\begin{array}{l}
\left.1-N_{1}\right) e^{-(\sqrt{\operatorname{Pr} N}) y}+N_{1} e^{-(\sqrt{S c K r}) y}+E c N_{5} e^{-(2 \lambda) y} \\
+E c N_{6} e^{-(2 \sqrt{\operatorname{Pr} N}) y}+E c N_{7} e^{-(2 \sqrt{S c K r}) y} \\
+E c N_{8} e^{-(\lambda+\sqrt{\operatorname{Pr} N}) y}+E c N_{9} e^{-(\sqrt{\operatorname{Pr} N}+\sqrt{S c K r}) y} \\
+E c N_{10} e^{-(\lambda+\sqrt{S c K r}) y} E c+E c A_{2} e^{-\sqrt{\operatorname{Pr} N} y}
\end{array}\right) \\
+\varepsilon e^{n t} \\
\left.+N_{2}\right) e^{-\sqrt{\operatorname{Pr}(N+n i)} y}+N_{2} e^{-\sqrt{S c(K r+n) y}} \\
+E c N_{20} e^{-(\sqrt{\lambda}+\sqrt{\lambda+n i}) y} \\
+E c N_{21} e^{-(\sqrt{\lambda}+\sqrt{\operatorname{Pr}(N+n i)}) y} \\
+E c N_{22} e^{-(\sqrt{\lambda}+\sqrt{S c(K r+n)}) y} \\
+E c N_{23} e^{-(\sqrt{\operatorname{Pr} N}+\sqrt{\lambda+n i}) y} \\
+E c N_{24} e^{-(\sqrt{\operatorname{Pr} N}+\sqrt{\operatorname{Pr}(N+n i)}) y} \\
+E c N_{25} e^{-(\sqrt{\operatorname{Pr} N}+\sqrt{S c(K r+n)}) y} \\
+E c N_{26} e^{-(\sqrt{S c K r}+\sqrt{\lambda+n i}) y} \\
+E c N_{27} e^{-(\sqrt{S c K r}+\sqrt{S c(K r+n)}) y} \\
+E c N_{28} e^{-(\sqrt{S c K r}+\sqrt{\operatorname{Pr}(N+n i)}) y} \\
+E c A_{5} e^{-\sqrt{\operatorname{Pr}(N+n i)} y} \\
\end{array}\right\}
$$

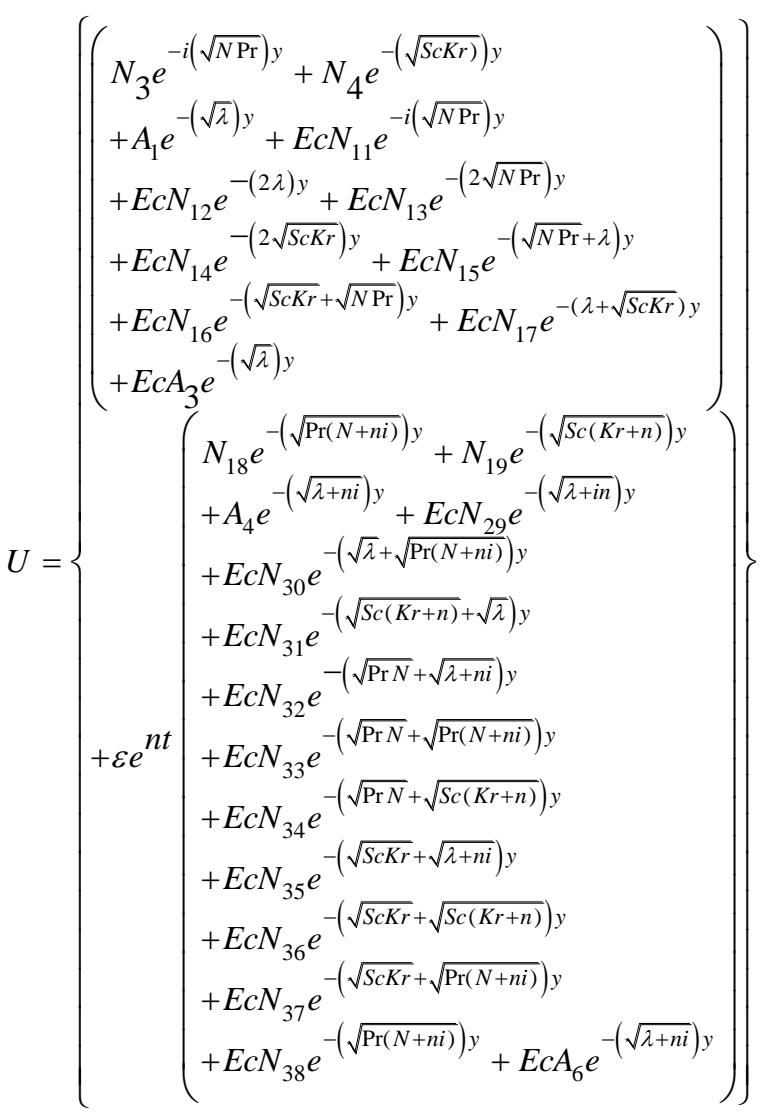

$$
\phi=e^{-(\sqrt{S c K r}) y}+\varepsilon e^{n t} e^{-(\sqrt{S c(K r+n)}) y}
$$

\subsection{Shear stress, Nusselt number and Sherwood number}

The Skin-friction at the plate, which in the non-dimensional form is given by

$$
C_{f}=\left[1+\frac{1}{\gamma}\right]\left[\frac{\tau_{w}}{\rho U_{0} V_{0}}\right]=\left[1+\frac{1}{\gamma}\right]\left[\frac{\partial u^{*}}{\partial y^{*}}\right]_{y=0}
$$

The rate of heat transfer coefficient, which in the nondimensional form in terms of the Nusselt number is given by

$$
N_{u}=-x \frac{\left[\frac{\partial T^{*}}{\partial y^{*}}\right]_{y=0}}{\left[T_{w}^{*}-T_{\infty}^{*}\right]} \Rightarrow N_{u} \operatorname{Re}_{x}^{-1}=-\left[\frac{\partial \theta}{\partial y}\right]_{y=0}
$$

The rate of mass transfer coefficient, which in the nondimensional form in terms of the Sherwood number, is given by

$S_{h}=-x \frac{\left[\frac{\partial C^{*}}{\partial y^{*}}\right]_{y=0}}{\left[C_{w}^{*}-C_{\infty}^{*}\right]} \Rightarrow S_{h} \operatorname{Re}_{x}^{-1}=-\left[\frac{\partial c}{\partial y}\right]_{y=0}$

\section{RESULTS AND DISCUSSION}

In the present study we have to select $\mathrm{t}=1.0, \mathrm{n}=0.5, \epsilon=0.03$, $\eta=0.003 \xi=0.03$ while $\beta, \mathrm{Kr}, \mathrm{Pr}, \mathrm{Gr}, \mathrm{Gm}, \gamma, \mathrm{M}, \mathrm{Ra} \mathrm{Ec}$, Sc, $\beta_{i}, \beta_{e}$, Dr and $\mathrm{R}$ are varied over a range, which listed in the figures. The variation in velocity and temperature profiles of $y$ for various values in Casson fluid parameter $\beta$ are shown in Figure 1 and Figure 15. Here we find that as the values of Casson fluid parameter $\beta$ increases it leads to decrees in velocity and increases in temperature. The variation in velocity profile with y for various values in Grashof numbers are shown in Figure 2. This figure reflects that with increase in $\mathrm{Gr}$ there is increase in fluid velocity due to improvement of the buoyancy force. The variation in velocity profile with y for different values in Modified Grashof number is shown in Figure 3. The modified Grashof number Gm defines the ratio of the species buoyancy force to the viscous hydrodynamic force. As expected, the fluid velocity increases as Grashof number $\mathrm{Gm}$ increases. The variety of velocity and concentration profiles with y for various esteems in chemical reaction parameter $K r$ is appeared in the Figure 2 and Figure 20 these figures mirror that with increment in $K r$ there is an increase in fluid velocity and diminish in concentration. Figure 5 and Figure 19. Here we find that as the values of permeability of the porous medium $\gamma$ increases it leads to decrease in velocity and diminish in temperature. The effect of Rotational parameter $R$ on the velocity and temperature profiles are shown in Figure 6 and Figure 18 velocity profiles decreases and temperature profile increases with an increase in $R$. The influence of Magnetic parameter on velocity profiles is as shown in the Figure 7 by keeping other parameters in rest. 
The nearness of magnetic field in an electrically conducting fluid presents a force called "Lorentz force" which acts against the flow if the magnetic field is connected in the normal direction as considered in the present issue. This sort of resistive force has a tendency to back off the flow field. Additionally, it is observed that the velocity of fluid reductions with expanding magnetic parameter. The variation in velocity profile with $y$ for various values in Ion-slip parameter is shown in Figure 8 and Figure 14. These figures reflect that with increase in Ion-slip parameter $\beta_{i}$ there is decrease in fluid velocity and temperature. The variations in velocity and temperature profiles with y for various values in Dufour number $D r$ are shown in Figure 9 and Figure 13 these figures reflect that with increase in Dr there is increase in fluid velocity and temperature. It is observed that in Figure 10 and Figure 16 the velocity and temperature decreases with increase in hall parameter $\beta_{e}$. Figure 11 depict the effect of $\mathrm{Ra}$ on the temperature profile. It is observed that temperature scores increases with the increase of thermal radiation parameter Ra. Figures 12 depict the effect of Eckert number $E c$ on the temperature profiles .It is revealed that temperature scores increases with the increasing of the Eckert number $E c$. Eckert number physically is a measure of frictional heat in the system. For different values of the Prandtl number $\mathrm{Pr}$, the Temperature profile is plotted in Figure 17 Here we find that as the values of Prandtl number $\mathrm{Pr}$ increase then the Temperature profile decreases. To be realistic, the numerical values of Prandtl number $\operatorname{Pr}$ are chosen as $\operatorname{Pr}=0.71$, and $\operatorname{Pr}=7.00$, which correspond to air and water at 20 degree Celsius's respectively. Figure 21 Here we find that as the values of Schmidt number $S c$ increase then it leads to the concentration decreases. The values of the Schmidt number are chosen to represent the presence of various species Hydrogen $(S c=0.22)$ Oxygen $(S c=0.60)$, Carbon dioxide $(S c=0.94)$, the values of other parameters are chosen arbitrarily. The numerical estimations of the rest of the parameters are picked selfassertively. Numerical values of the Skin-friction coefficient $\tau_{w}$ Nusselt number $N_{u}$ Sher wood number $S_{h}$ are shown below. From the Table 1 depict the effect of the Prandtl number $\operatorname{Pr}$ Schmidt number $S c$, hall parameter $\beta_{e}$, Ion-slip parameter $\beta_{i}$ Eckert number $(E c)$, thermal radiation absorption $(R a)$, chemical reaction $(K r)$, Porous medium $\gamma$ rotational parameter $R$, Dufour effect $D u$, Casson fluid parameter $\beta$ on the Skin friction coefficient $\tau_{w}$ and Nusselt number $N_{w}$ respectively. It is observed that from the table the parameters $D r, G r, G m, E c$ and $R a$ increases then it leads to increases in skin-friction and Nusselt number. If the parameters $K r, M, \gamma$ and $\beta$ are increases then the corresponding values of Skin friction coefficient $\tau_{w}$ and Nusselt number $N_{w}$ decreases. But ion-slip parameter $\beta_{i}$ and $\operatorname{Pr}$ increases then it leads to skin friction increases and Nusselt number decreases. However, $\beta_{e}$, $R$ and $S c$ increases then the corresponding values of Skin friction coefficient $\tau_{w}$ decreases and Nusselt number $N_{w}$ increases. From the Table 2 shows that the effect of the Schmidt number Sc and chemical reaction $K r$ for different values on Sherwood $S_{h}$. It is observed from the table that as $S c$ and $K r$ increases the Sherwood decreases.

\section{VALIDATION OF THE RESULTS}

In order to assess the accuracy of our method, we have compared our results with accepted data sets for the velocity distribution for a case of Transfer in unsteady Magneto Hydrodynamic Casson fluid flow with convective boundary conditions by Pushpalatha et al. [11], in the absence of Eckert number (Ec), thermal radiation (Ra) and hall and ion-slip current effect by taking different values for Casson fluid parameter and Chemical reaction and Porous medium keeping the other parameters fixed and these results are presented in Figure 1 and Figure 4. The results of this comparison are found to be in good agreement.

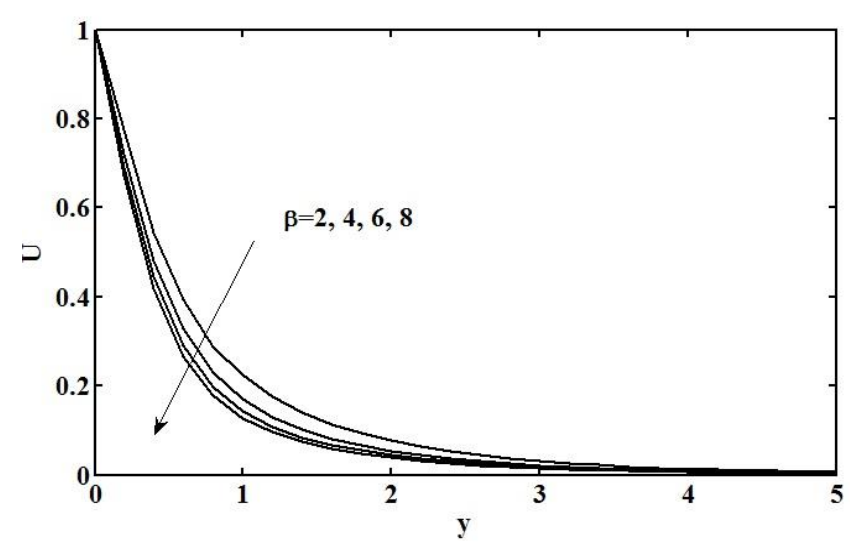

Figure 1. Effect of $\beta$ for different values on Velocity

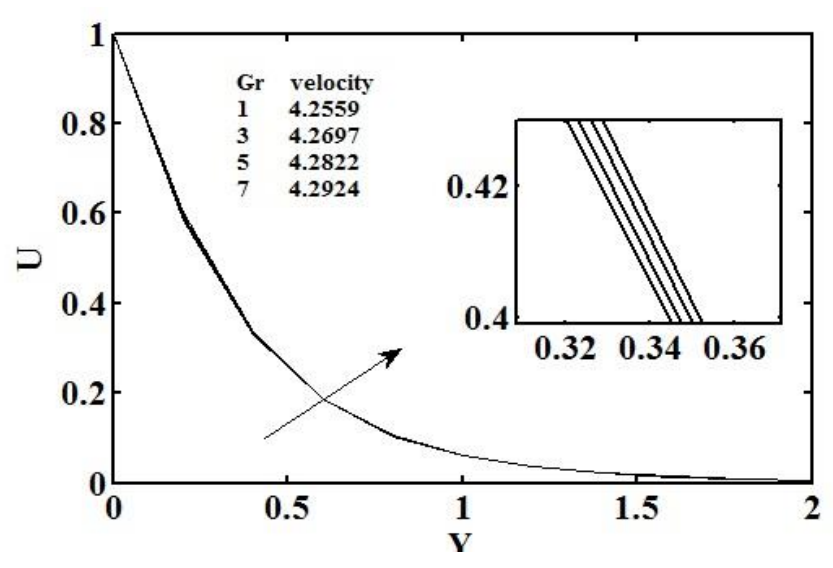

Figure 2. Effect of Gr for different values on Velocity

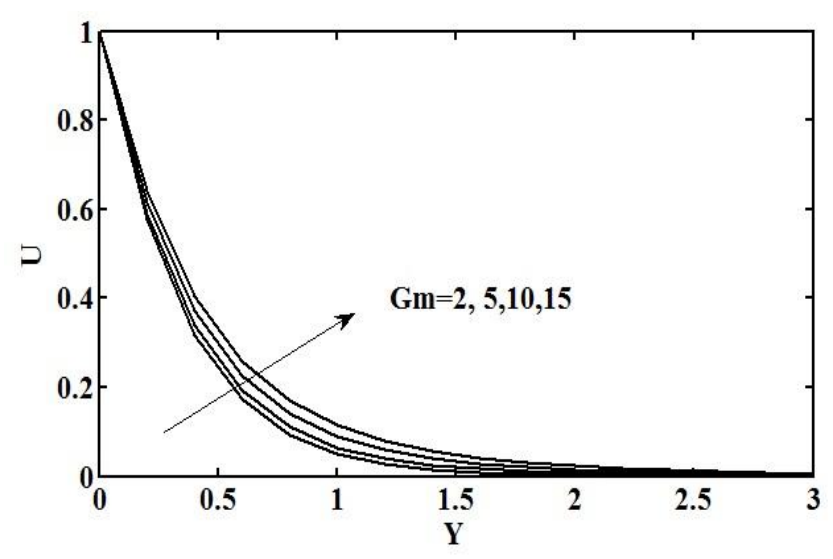

Figure 3. Effect of Gm for different values on Velocity 


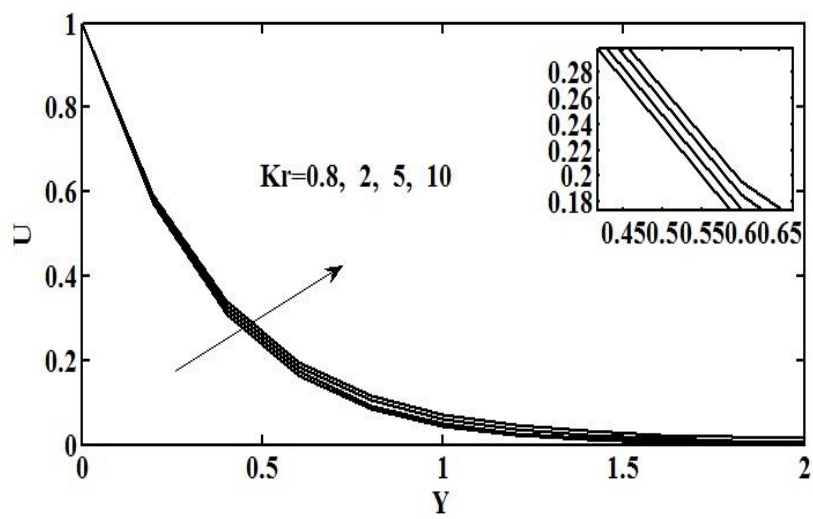

Figure 4. Effect of $\mathrm{Kr}$ for different values on Velocity

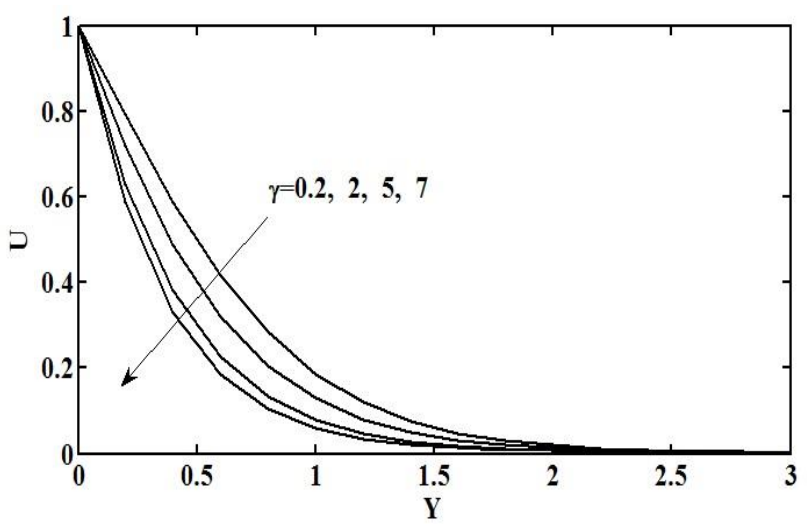

Figure 5. Effect of $\gamma$ for different values on Velocity

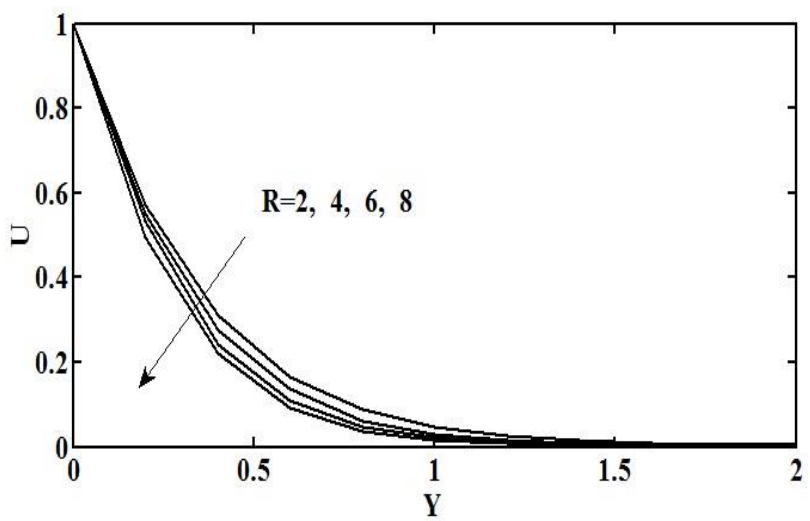

Figure 6. Effect of R for different values on Velocity

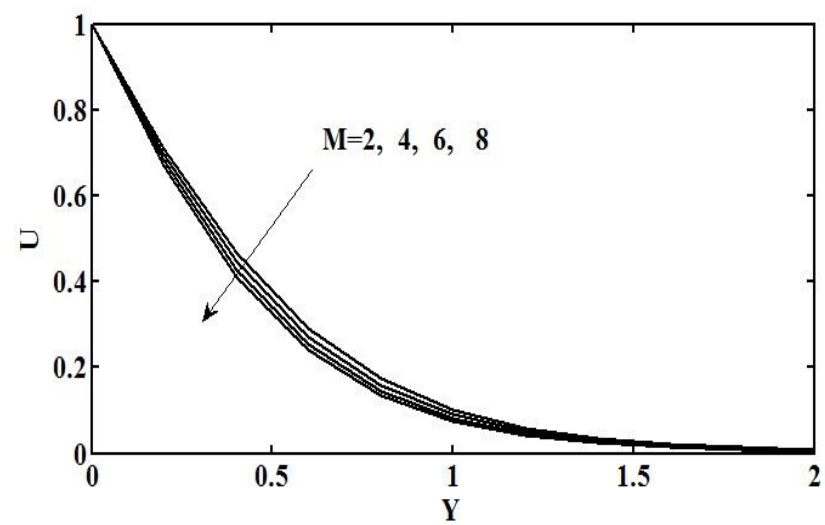

Figure 7. Effect of $M$ for different values on Velocity

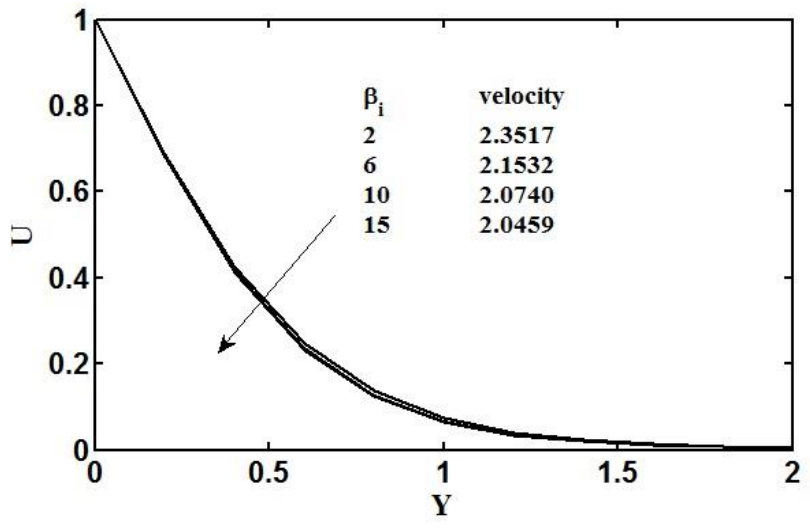

Figure 8. Effect of Dr for different values on Velocity

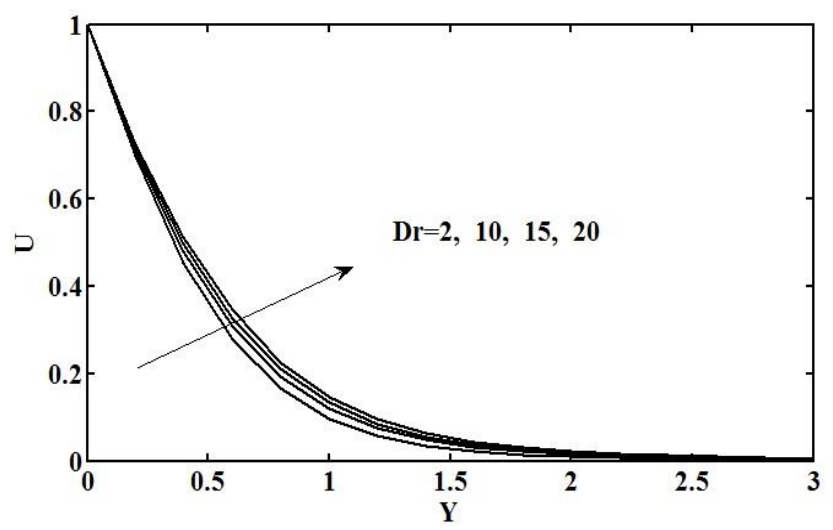

Figure 9. Effect of Dr for different values on Velocity

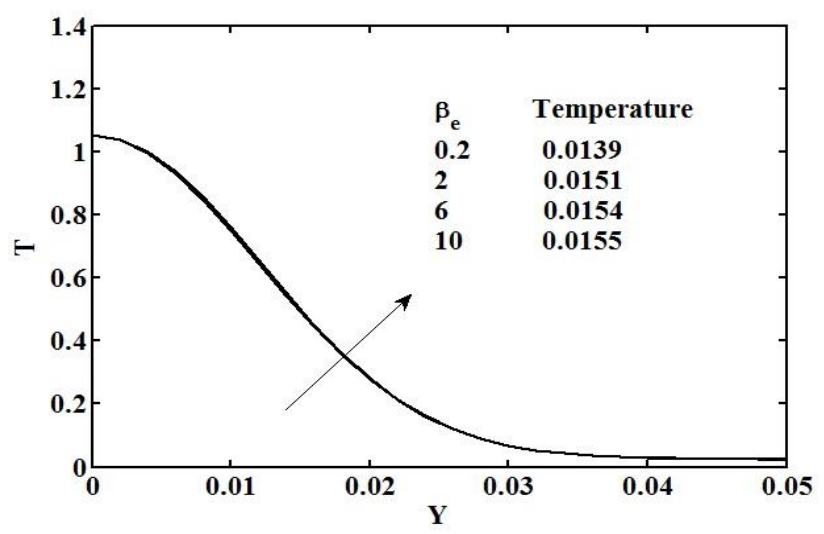

Figure 10. Effect of $\beta_{e}$ for different values on Velocity

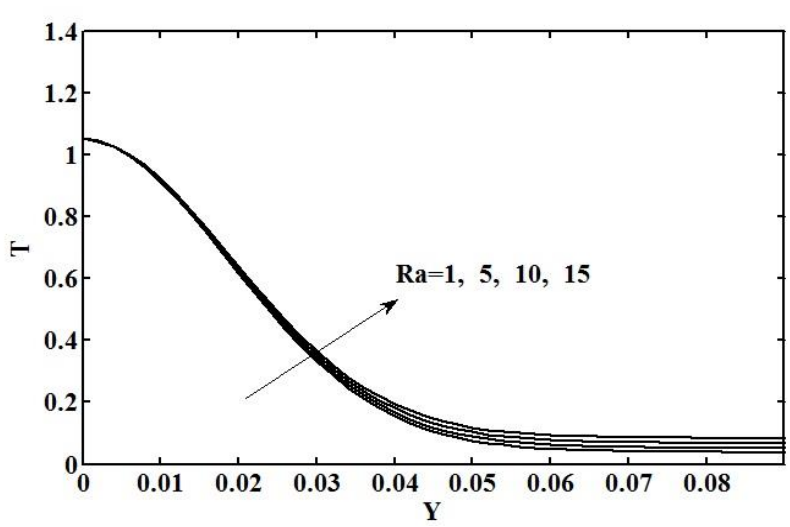

Figure 11. Effect of Ra for different values on Velocity 


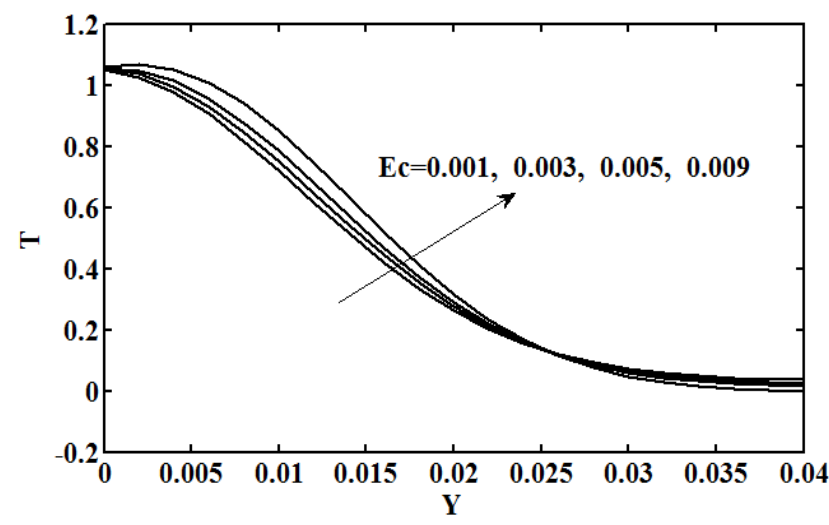

Figure 12. Effect of Ec for different values on Temperature

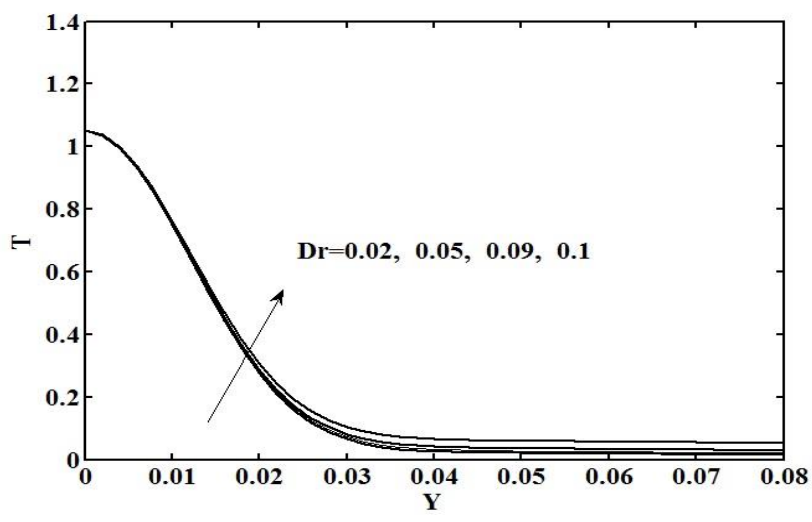

Figure 13. Effect of Dr for different values on Temperature

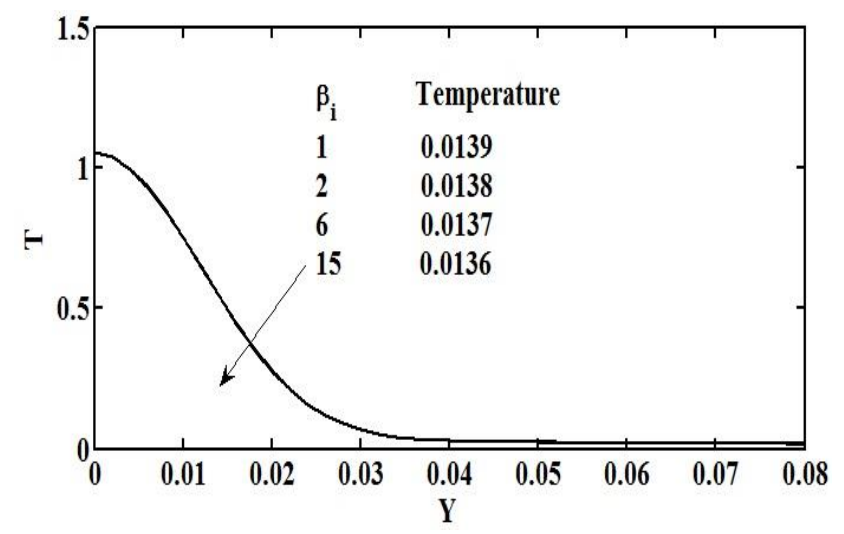

Figure 14. Effect of $\beta_{i}$ Dr for different values on

Temperature

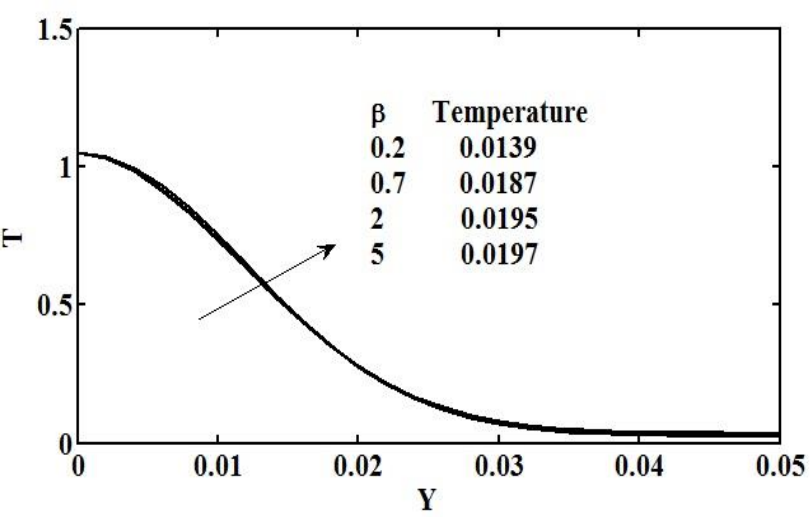

Figure 15. Effect of $\beta$ for different values on Temperature

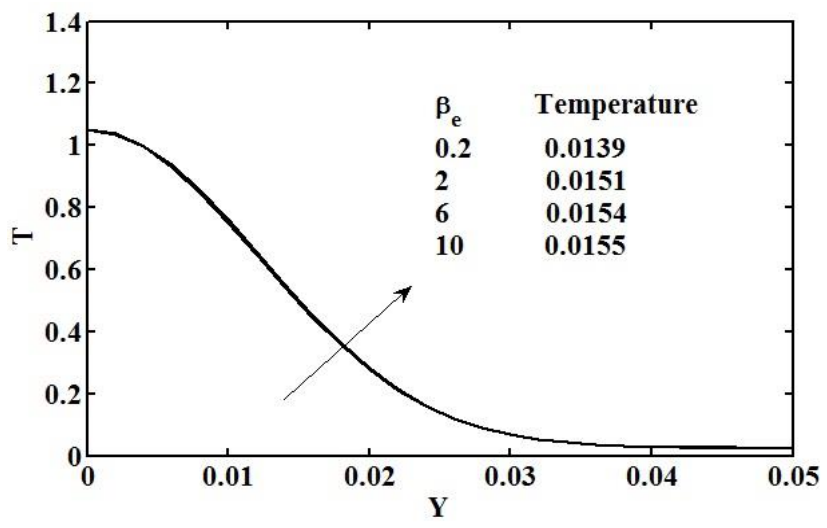

Figure 16. Effect of $\beta_{e}$ for different values on Temperature

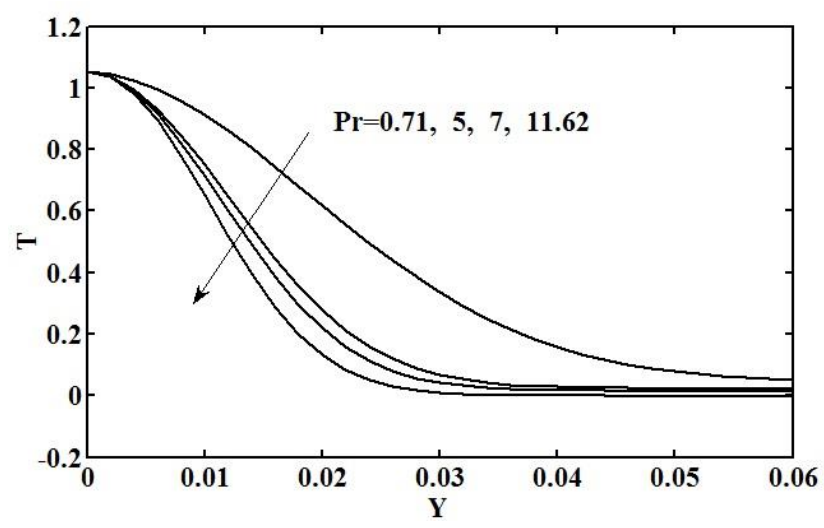

Figure 17. Effect of Pr for different values on Temperature

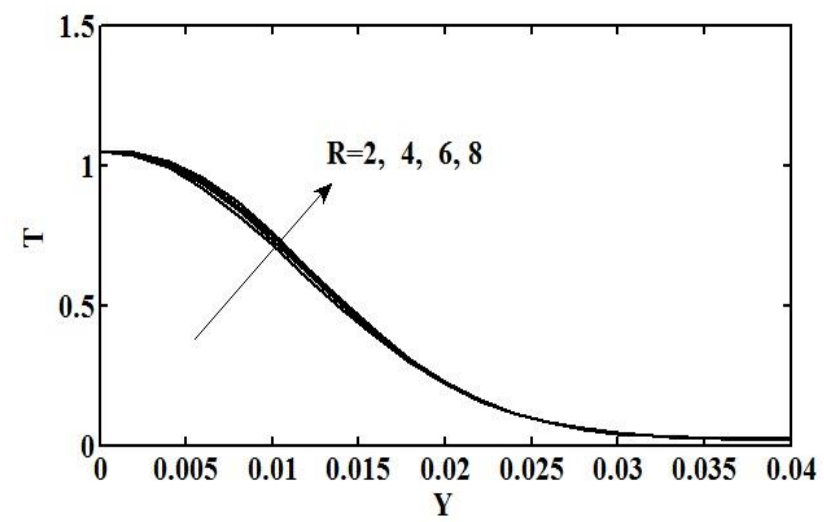

Figure 18. Effect of $R$ for different values on Temperature

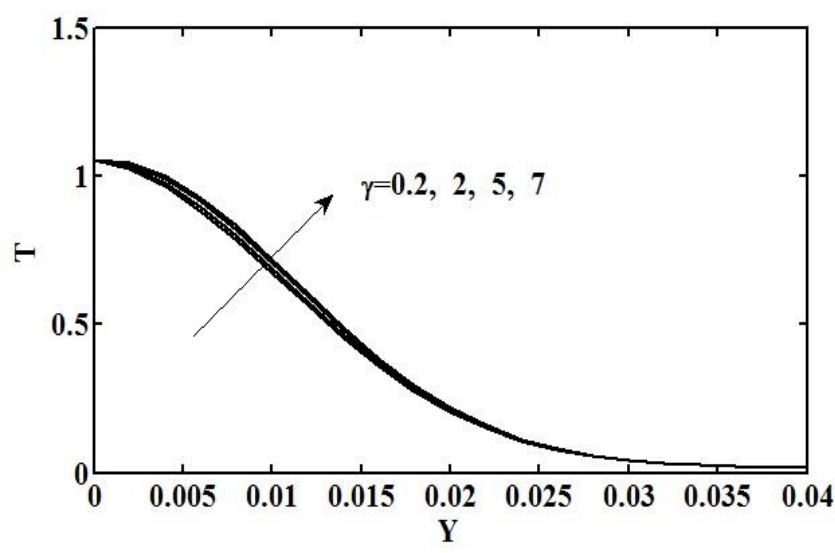

Figure 19. Effect of $\gamma$ Dr for different values on Temperature 
Table 1. Effect of Pr, Dr, Gm, GM, $\beta, \beta_{\mathrm{e}}, \beta_{\mathrm{e}}, \gamma, \mathrm{Kr}, \mathrm{M}, \mathrm{R}, \mathrm{Sc}, \mathrm{Ec}$ and Ra on Skin friction $\tau_{w}$ and Nussle number $N_{w}$

\begin{tabular}{|c|c|c|c|c|c|c|c|c|c|c|c|c|c|c|c|}
\hline $\operatorname{Pr}$ & Dr & $\mathrm{Gm}$ & $\mathrm{Gr}$ & $\beta$ & $\beta_{\mathrm{e}}$ & $\beta_{\mathrm{i}}$ & $\gamma$ & $\mathrm{Kr}$ & $\mathrm{M}$ & $\mathrm{R}$ & $\mathrm{Sc}$ & $\mathrm{Ec}$ & $\mathrm{Ra}$ & $\tau_{w}$ & $N_{w}$ \\
\hline 5 & 0.2 & 4 & 2 & 0.2 & 0.2 & 0.2 & 2 & 2 & 2 & 2 & 0.61 & 0.003 & 1 & -5.6582 & -302.2987 \\
\hline 7 & & & & & & & & & & & & & & -5.4953 & -352.3593 \\
\hline \multirow[t]{40}{*}{11.62} & & & & & & & & & & & & & & -5.1165 & -443.8403 \\
\hline & 0.02 & & & & & & & & & & & & & -5.6598 & -316.7142 \\
\hline & 0.05 & & & & & & & & & & & & & -5.6595 & -314.3116 \\
\hline & 0.09 & & & & & & & & & & & & & -5.6592 & -311.1082 \\
\hline & & 2 & & & & & & & & & & & & -5.7013 & -302.3444 \\
\hline & & 5 & & & & & & & & & & & & -5.6367 & -302.2763 \\
\hline & & 10 & & & & & & & & & & & & -5.5291 & -302.1679 \\
\hline & & & 1 & & & & & & & & & & & -5.8789 & -302.2997 \\
\hline & & & 3 & & & & & & & & & & & -5.4376 & -302.2977 \\
\hline & & & 5 & & & & & & & & & & & -4.9963 & -302.2957 \\
\hline & & & & 0.2 & & & & & & & & & & -5.6582 & -302.2987 \\
\hline & & & & 0.7 & & & & & & & & & & -9.0554 & -304.8165 \\
\hline & & & & 2 & & & & & & & & & & -11.5835 & -305.4107 \\
\hline & & & & & 0.7 & & & & & & & & & -5.9423 & -297.9718 \\
\hline & & & & & 2 & & & & & & & & & -6.2008 & -293.8865 \\
\hline & & & & & 6 & & & & & & & & & -6.3738 & -291.0776 \\
\hline & & & & & & 2 & & & & & & & & -5.6100 & -303.0167 \\
\hline & & & & & & 6 & & & & & & & & -5.5541 & -303.8416 \\
\hline & & & & & & 10 & & & & & & & & -5.5259 & -304.2542 \\
\hline & & & & & & & 0.2 & & & & & & & -5.5104 & -304.4802 \\
\hline & & & & & & & 2 & & & & & & & -5.6582 & -302.2987 \\
\hline & & & & & & & 5 & & & & & & & -5.8972 & -298.6710 \\
\hline & & & & & & & & 2 & & & & & & -5.6582 & -302.2987 \\
\hline & & & & & & & & 3 & & & & & & -5.6620 & -297.6543 \\
\hline & & & & & & & & 4 & & & & & & -5.6582 & -292.8946 \\
\hline & & & & & & & & & 4 & & & & & -5.8946 & -298.7100 \\
\hline & & & & & & & & & 6 & & & & & -6.1228 & -295.1338 \\
\hline & & & & & & & & & 8 & & & & & -6.3436 & -291.5727 \\
\hline & & & & & & & & & & 1 & & & & -4.1148 & -322.4672 \\
\hline & & & & & & & & & & 4 & & & & -7.9484 & -262.9497 \\
\hline & & & & & & & & & & 6 & & & & -9.7458 & -225.8581 \\
\hline & & & & & & & & & & & 0.22 & & & -5.6513 & -308.0764 \\
\hline & & & & & & & & & & & 0.42 & & & -5.6553 & -305.1356 \\
\hline & & & & & & & & & & & 0.61 & & & -5.6582 & -302.2987 \\
\hline & & & & & & & & & & & & 0.001 & & -5.9478 & -326.1582 \\
\hline & & & & & & & & & & & & 0.003 & & -5.6582 & -302.2987 \\
\hline & & & & & & & & & & & & 0.005 & & -5.3687 & -278.4392 \\
\hline & & & & & & & & & & & & & 1 & -5.6582 & -302.2987 \\
\hline & & & & & & & & & & & & & 5 & -5.6552 & -273.4387 \\
\hline & & & & & & & & & & & & & 10 & -5.6513 & -237.3637 \\
\hline
\end{tabular}

Table 2. Effect of Sc and Kr on Sherwood numbers $S_{h}$

\begin{tabular}{|c|c|c|}
\hline $\mathrm{Sc}$ & $\mathrm{Kr}$ & $S_{h}$ \\
\hline 0.22 & 5 & -1.0653 \\
\hline 0.42 & 5 & -1.4719 \\
\hline 0.61 & 5 & -1.7739 \\
\hline 0.81 & 5 & -2.0441 \\
\hline 0.61 & 2 & -1.1231 \\
\hline 0.61 & 3 & -1.3747 \\
\hline 0.61 & 4 & -1.5869 \\
\hline 0.61 & 5 & -1.7739 \\
\hline
\end{tabular}




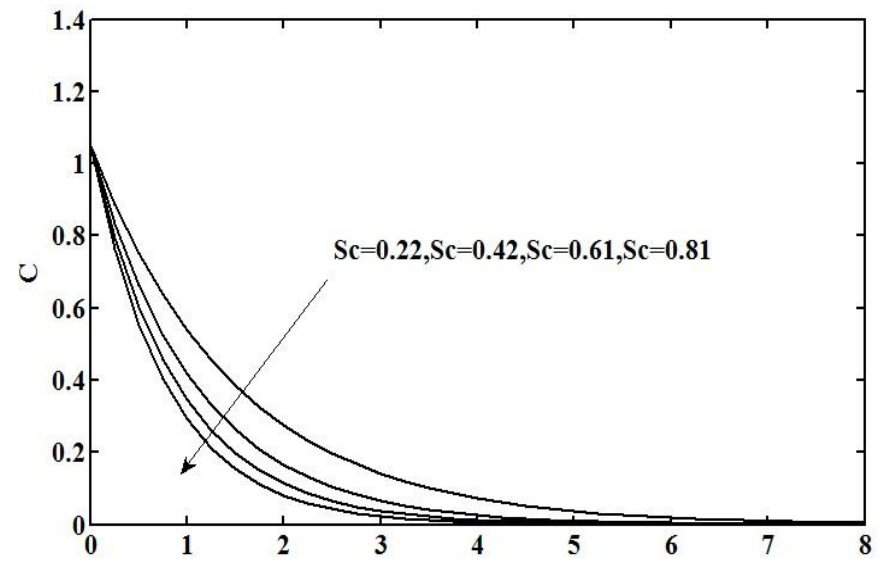

Figure 20. Effect of Sc for different values on Concentration

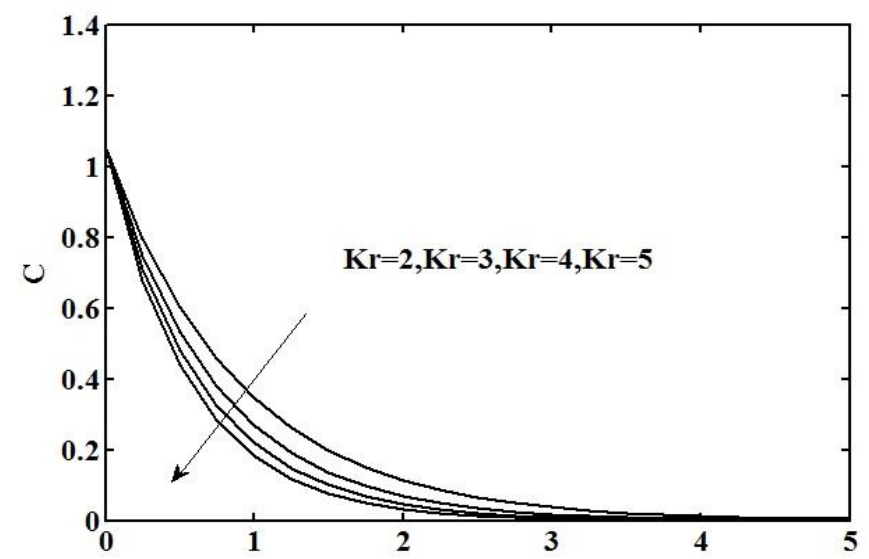

Figure 21. Effect of $\mathrm{Kr}$ for different values on Concentration

\section{CONCLUSIONS}

- Temperature profiles increases with the increase of Thermal radiation parameter $\mathrm{Ra}$

- Concentration profiles decrease near the plate with the increase of chemical reaction parameter $K r$ and Schmidt number $S c$.

- Temperature profile increases with the increase of Eckert number $E c$.

- Velocity decreases with increase in Magnetic field parameter $M$.

- As the Ion-slip parameter $\beta_{i}$ increases the Velocity and temperature profiles decreases but the velocity and temperature increase with increase of Hall current parameter $\beta_{e}$.

- As the Dufour effect parameter $D r$ increases the velocity and temperature increases

- As the porous parameter $\gamma$ increases the velocity and temperature decreases.

- As the Casson fluid parameter increases then velocity decreases and temperature increases.

\section{ACKNOWLEDGMENTS}

The authors express their gratitude to the referees for their constructive review of the paper and for valuable comments.

\section{REFERENCES}

[1] Biswas, Mondal, Sarkar and Ahmmed. (2017). Effects of radiation and chemical reaction on MHD unsteady heat and mass transfer of Casson fluid flow past a vertical plate. Journal of Advances in Mathematics and Computer Science 23(2): 1-16.

[2] Reddy GJ, Raju RS, Rao JA. (2017). Influence of viscous dissipation on unsteady MHD natural convective flow of Casson fluid over an oscillating vertical plate. Ain Shams Engineering Journal 5(1): 1-9.

[3] Attia HA. (2010). Transient MHD Couette flow of a Casson fluid between parallel plates with heat transfer. Italian Journal of Pure and Applied Mathematics 27: 1938.

[4] Raju RS, Anita JR. (2017). MHD Casson viscous dissipative fluid flow past a vertically inclined plate in presence of heat and mass transfer. Frontiers in Heat and Mass Transfer. 8(27): 1-12.

[5] Saidulu N, Lakshman AV. (2016). MHD flow of Casson fluid with slip effects over an exponentially porous stretching sheet in presence of thermal radiation, viscous dissipation and heat source/sink. American Research J. of Mathematics 2(1): 1-15.

[6] Raju RS, Reddy BM, Reddy GJ. (2017). Influence of angle of inclination on unsteady MHD Casson fluid flow past a vertical surface filled by porous medium in presence of constant heat flux, chemical reaction and viscous dissipation. J of Nano Fluids 6(4): 668-679.

[7] Hall E. (1979). On a new action of the magnet on electric currents. American J. Math. 2: 287-292.

[8] Anika N, Hoque M, Hossain I. (2015). Thermal diffusion effect on unsteady viscous MHD microplar fluid flow through an infinite vertical plate with hall and ion-slip current. Procedia Engineering. 105: 160-166.

[9] Srinivasacharya, Shafeeurrahaman. (2017). Mixed convection flow of Nanofluid in a vertical channel with hall and ion-slip effects. Frontiers in Heat and Mass Transfer 8(11): 1-8.

[10] Srinivasacharya, Kaladhar. (2013). Analytical solution for hall and ion-slip effects on mixed convection flow of couple stress fluid between parallel disks. Mathematical and Computer Modeling 57: 2494-2509.

[11] Pushpalatha K, Sugunamma V, Reddy JVR, Sandeep N. (2016). Heat and Mass transfer in unsteady MHD Casson fluid flow with convective boundary conditions. Int. J. of Advanced Science and Technology 91: 19-38.

[12] Pannerselvi, Kowsalya. (2015). Ion slip and Dufour effect on unsteady free convection flow past an infinite vertical plate with oscillatory suction velocity and variable permeability. International Journal of Science and Research 4(12): 2079-2090.

[13] Abuga, Kinyanju, Sigey. (2011). An investigation of the effect of Hall currents and rotational parameter on dissipative fluid flow past a vertical semi-infinite plate. J. Eng. Tech. Res. 3: 314-320.

[14] Ojjela, Naresh Kumar. (2014). Hall and Ion slip effects on free convection heat and mass transfer of chemically reacting couple stress fluid in a porous expanding or contracting walls with soret and Dufour effects. Frontiers in Heat and Mass Transfer 5(22): 1-12.

[15] Katarina H, Patel HR. (2016). Radiation and chemical reaction effects on MHD Casson fluid flow past an 
oscillating vertical plate embedded in porous medium. Alexandria Engineering Journal 55: 583-595.

[16] Shateyi, Motsa, Sibanda, (2010). The effects of thermal radiation, hall currents, soret, and Dufour on MHD flow by mixed convection over a vertical surface in porous media. Math. Probl. Eng. 1-20.

[17] Sreedevi, Rao R, Rao P, Chamkha. (2016). Combined influence of radiation absorption and Hall current effects on MHD double-diffusive free convective flow past a stretching sheet. Ain Shams Engineering Journal. 7: 383397.

[18] Gireesha, Mahanthesh. (2013). Perturbation solution for radiating viscoelastic fluid flow and heat transfer with convective boundary condition in non-uniform channel with Hall current and chemical reaction. Hindawi Publishing Corporation ISRN Thermodynamics 1-14.

[19] Delowar, Hossain, Samad A, Alam M. MHD free convection and mass transfer flow through a vertical oscillatory porous plate with Hall, Ion-slip currents and heat source in a rotating system. Procedia Engineering 105: 56-63.

[20] Mythili D, Siva RRAJ, Rashid MM. (2017). Heat generating/absorbing and chemically reacting Casson fluid flow over a vertical cone and flat plate saturated with non-Darcy porous medium. International Journal of Numerical Methods for Heat and Fluid Flow 27: 156173.

[21] Benazir J, Siva Raj, Makinde, (2016). unsteady MHD Casson fluid flow over a vertical cone and flat plate with non-uniform heat source/sink. International Journal of Eng. Research in Africa 21: 69-83.

[22] Raju RS. (2017). Transfer effects on an unsteady MHD mixed convective flow past a vertical plate with chemical reaction. Engineering Transactions Eng. Trans. 65(2): 221-249.

[23] Salem AM, Abd El-Aziz M. (2008). Effect of Hall currents and chemical reaction on Hydro Magnetic flow of a stretching vertical surface with internal heat generation/absorption. Applied Mathematical Modeling 32(7): 1236-1254.

[24] Sudhakar K, Srinivasa RR, Rangamma M. (2013). Hall effect on an unsteady MHD flow past along a porous flat plate with thermal diffusion, diffusion thermo and chemical reaction. Journal of Physical and Mathematical Sciences 4(1): 370-395.

[25] Srinivasa RR. (2016). Combined influence of thermal diffusion and diffusion thermo on unsteady Hydro Magnetic free convective fluid flow past an infinite vertical porous plate in presence of chemical reaction. Journal of Institution of Engineers (India), Series C 1-11.

[26] Raju S, Reddy M, Rashidi, Gorla R.S.R., (2016). Application of finite element method to unsteady MHD free convection flow past a vertically inclined porous plate including thermal diffusion and diffusion thermo effects. Journal of Porous Media 19(8): 701-722.

[27] Srinivasa RR, Reddy J, Rao A, Rashidi, Gorla RSR. (2016). Analytical and Numerical study of unsteady MHD free convection flow over an exponentially moving vertical plate with heat absorption. International Journal of Thermal Sciences 107: 303-315.

\section{NOMENCLATURE}

B

$B_{0}$

$C_{w}$

$C_{\infty}$

C

$C_{p}$

$D_{m}$

Ec

$\mathrm{Gr}$

$\mathrm{Gr}$

$g$

J

$\mathrm{k}$

$K_{r}$

$n^{*}$

Pr

$p_{y}$

$q_{r}^{*}$

$Q_{0}$

$\mathrm{R}$

$R a$

$\mathrm{Sc}$

$S_{h}$

$t^{*}$

$T_{w}$

$T_{\infty}$

$U_{0}$

$\mathrm{U}$

$u$

$\mathrm{x}, \mathrm{y}$

\section{Greek symbols}

$\gamma$

$\Omega$

$\eta$

$\tau_{w}$

$\xi$

$\beta$

$\theta$

$\mu_{B}$

$\beta_{e}$

$\beta_{i}$

$\psi$

$\psi^{*}$

$\alpha$

1)
Magnetic field

Magnetic component (A. $\left.m^{-1}\right)$

Concentration at the plate $\left(\mathrm{kg} . \mathrm{m}^{-1}\right)$

Concentration outside of the plate $\left(\mathrm{kg}^{\mathrm{m}} \mathrm{m}^{-3}\right)$

Dimensionless fluid concentration

Specific heat at constant pressure $\left(J . \mathrm{kg}^{-1} \cdot \mathrm{K}\right)$

Co-efficient of mass diffusivity

Eckret number

Grashof number

Modified Grashof number

acceleration due to $\operatorname{gravity}\left(m \cdot S^{-2}\right)$

Current density

Magnetic permeability of the porous medium

Chemical reaction parameter $\left(m \cdot S^{-1}\right)$

Mining

Prandtl number

Yield tress fluid

Radiation heat flux density $\left(W . m^{-2}\right)$

Heat absorption quantity

Rotational parameter

Thermal radiation parameter

Schmidt number

Sherwood numbers

Dimensional time

Temperature at the plate $(K)$

Temperature outside of the boundary lyre $(K)$

Uniform velocity

Dimensionless primary velocity

components of velocity vector in $\mathrm{x}$ $\operatorname{direction}\left(\mathrm{m} . \mathrm{S}^{-1}\right)$

Cartesian co-ordinates

Permeability of porous medium

Rotational velocity component $(\mathrm{Hz})$

Radiation parameter

Skin-friction coefficient

Heat source parameter

Casson fluid parameter

Temperature $(k)$

plastic dynamic viscosity $\left(\mathrm{Nsm}^{-1}\right)$

Hall parameter

Ion-slip parameter

Thermal expansion co-efficient $\left(\mathrm{K}^{-1}\right)$

Concentration expansion co-efficient $\left(\mathrm{m}^{3} . \mathrm{kg}\right.$

Heat source parameter 


$\begin{array}{ll}\vartheta & \text { Kinematic velocity }\left(\mathrm{m}^{2} \cdot \mathrm{S}^{-1}\right) \\ \rho & \text { Density of the fluid }\left(\mathrm{kg} \cdot \mathrm{m}^{-3}\right) \\ \sigma_{\rho} & \text { Electrical conductivity }\left(\Omega^{-1} \mathrm{~m}^{-1}\right) \\ \sigma & \text { Thermal conductivity }\left(\mathrm{m}^{2} \cdot \mathrm{S}^{-1}\right) \\ \varepsilon & \text { Arbitrary constant } \\ N_{u} & \text { Nusselt number } \\ \alpha_{e} & =1+\beta_{e} \beta_{i} \text { constant(Hall and ion slip } \\ \mu & \text { parameters }) \\ P & \text { Density of the fluid } \\ * & \text { Plate } \\ W & \text { Dimensionless properties } \\ \infty & \text { Wall condition } \\ & \text { Free stream condition }\end{array}$

\section{APPENDIX}

$\mathrm{p}=\sqrt{\frac{\lambda}{1+\frac{1}{\beta}}}, \mathrm{q}=\sqrt{\frac{\lambda+n i}{1+\frac{1}{\beta}}} a=\sqrt{S c K r}, b=\sqrt{S c(K r+n)}$

$e=\sqrt{\operatorname{Pr} N}, f=\sqrt{\operatorname{Pr}(N+n i)}, N_{1}=\frac{-R a \operatorname{Pr}-\text { Dra }^{2}}{\Gamma a^{2}+\xi}$

$N_{2}=\frac{-R a \operatorname{Pr}-b^{2} \operatorname{Pr} D r}{b^{2}-\operatorname{Pr}(N+n i)}$

$N_{3}=\frac{-G r\left(1-N_{1}\right)}{e^{2}-\lambda}$

$N_{4}=\frac{-\left(G r N_{1}+G m\right)}{e^{2}-\lambda}$

$N_{5}=\frac{-\operatorname{Pr}\left[1+\frac{1}{\beta}\right] \lambda^{2} A_{1}^{2}}{4 \lambda^{2}-\operatorname{Pr} N}$

$N_{6}=\frac{-\operatorname{Pr}\left[1+\frac{1}{\beta}\right] e^{2} N_{3}^{2}}{4 e^{2}-\operatorname{Pr} N}$

$N_{7}=\frac{-\operatorname{Pr}\left[1+\frac{1}{\beta}\right] N_{4}^{2} a^{2}}{4 a^{2}-\operatorname{Pr} N}$

$N_{8}=\frac{-2 e \operatorname{Pr}\left[1+\frac{1}{\beta}\right] \lambda A_{1}}{(e+\lambda)^{2}-\operatorname{Pr} N}$

$N_{9}=\frac{-2 a e \operatorname{Pr}\left[1+\frac{1}{\beta}\right] N_{4} N_{3}}{(e+a)^{2}-\operatorname{Pr} N} N_{10}=\frac{-2 \operatorname{Pr}\left[1+\frac{1}{\beta}\right] a \lambda A_{1} N_{4}}{(\lambda+a)^{2}-\operatorname{Pr} N}$

$A_{1}=\left(1-N_{3}-N_{4}\right)$

$A_{2}=\left(1-N_{5}-N_{6}-N_{7}-N_{8}-N_{9}-N_{10}\right)$

$N_{11}=\frac{-G r A_{2}}{e^{2}-\lambda}$,

$$
\begin{aligned}
& N_{12}=\frac{-G r N_{4}}{4 \lambda^{2}-\lambda}, \\
& N_{13}=\frac{-G r N_{5}}{4 e^{2}-\lambda} \\
& N_{14}=\frac{-G r N_{6}}{4 a^{2}-\lambda} \\
& N_{15}=\frac{-G r N_{7}}{(\lambda+e)^{2}-\lambda}
\end{aligned}
$$$$
N_{16}=\frac{-G r N_{8}}{(e+a)^{2}-\lambda}
$$$$
N_{17}=\frac{-G r N_{9}}{(\lambda+a)^{2}-\lambda}
$$$$
N_{18}=\frac{-G r\left(1-N_{2}\right)}{f^{2}-(\lambda+n i)}
$$$$
N_{19}=\frac{-G r N_{2}-G m}{b^{2}-(\lambda+n i)}
$$$$
N_{20}=\frac{-2 \operatorname{Pr}\left[1+\frac{1}{\beta}\right] A_{1} p g A_{5}}{(p+q)^{2}-\operatorname{Pr}(N+n i)}
$$$$
N_{21}=\frac{-2 \operatorname{Pr}\left[1+\frac{1}{\beta}\right] A_{1} p N_{18} f}{(p+f)^{2}-\operatorname{Pr}(N+n i)}
$$$$
N_{22}=\frac{-2 \operatorname{Pr}\left[1+\frac{1}{\beta}\right] N_{19} b A_{1} p}{(p+b)^{2}-\operatorname{Pr}(N+n i)}
$$$$
N_{23}=\frac{-2 \operatorname{Pr}\left[1+\frac{1}{\beta}\right] e q N_{3} A_{5}}{(e+q)^{2}-\operatorname{Pr}(N+n i)}
$$$$
N_{24}=\frac{-2 \operatorname{Pr}\left[1+\frac{1}{\beta}\right] e f N_{3} N_{18}}{(e+f)^{2}-\operatorname{Pr}(N+n i)}
$$$$
N_{25}=\frac{-2 \operatorname{Pr}\left[1+\frac{1}{\beta}\right] e b N_{3} N_{19}}{(e+b)^{2}-\operatorname{Pr}(N+n i)}
$$$$
N_{26}=\frac{-2 \operatorname{Pr}\left[1+\frac{1}{\beta}\right] a q N_{4} A_{5}}{(a+q)^{2}-\operatorname{Pr}(N+n i)}
$$$$
N_{27}=\frac{-2 \operatorname{Pr}\left[1+\frac{1}{\beta}\right] N_{4} b_{a N_{19}}}{(a+b)^{2}-\operatorname{Pr}(N+n i)}
$$$$
N_{28}=\frac{-2 \operatorname{Pr}\left[1+\frac{1}{\beta}\right] N_{4} a f N_{18}}{(a+f)^{2}-\operatorname{Pr}(N+n i)}
$$$$
N_{29}=\frac{-G r N_{20}}{(p+q)^{2}-(\lambda+n i)},
$$$$
N_{30}=\frac{-G r N_{21}}{(p+f)^{2}-(\lambda+n i)}
$$ 


$$
\begin{aligned}
& N_{31}=\frac{-G r N_{22}}{(p+b)^{2}-(\lambda+n i)} \\
& N_{32}=\frac{-G r N_{23}}{(e+f)^{2}-(\lambda+n i)} \\
& N_{33}=\frac{-G r N_{24}}{(e+f)^{2}-(\lambda+n i)}, \\
& N_{34}=\frac{-G r N_{25}}{(e+b)^{2}-(\lambda+n i)} \\
& N_{35}=\frac{-G r N_{26}}{(a+q)^{2}-(\lambda+n i)} \\
& N_{36}=\frac{-G r N_{27}}{(a+b)^{2}-(\lambda+n i)}
\end{aligned}
$$$$
N_{37}=\frac{-G r N_{28}}{(a+f)^{2}-(\lambda+n i)}
$$$$
N_{38}=\frac{-G r A_{5}}{f^{2}-(\lambda+n i)}
$$$$
A_{3}=-\left(N_{11}+N_{12}+N_{13}+N_{14}+N_{15}+N_{16}+N_{17}\right)
$$$$
A_{4}=-\left(N_{18}+N_{19}\right)
$$$$
A_{5}=-\left(N_{20}+N_{21}+N_{22}+N_{23}+N_{24}+N_{25}+N_{26}\right.
$$$$
\begin{array}{r}
A_{6}=-\left(N_{29}+N_{30}+N_{31}+N_{32}+N_{33}+N_{34}+N_{35}\right. \\
\left.+N_{36}+N_{37}+N_{38}\right)
\end{array}
$$ 